CONJUNTOS CONTROLÁVEIS: UMA ABORDAGEM DE SISTEMAS DINÂMICOS

PARA A TEORIA DO CONTROLE

Roberto Cáceres Anco

DISSERTAÇÃO APRESENTADA

$\mathrm{AO}$

INSTITUTO DE MATEMÁTICA E ESTATÍSTICA

DA

UNIVERSIDADE DE SÃO PAULO

PARA

OBTENÇÃO DO GRAU

DE

MESTRE EM MATEMÁTICA APLICADA

Área de Concentração: Matemática Aplicada

Orientador: Prof. Dr. Pedro Aladar Tonelli

- São Paulo, dezembro de 1996 - 


\title{
CONJUNTOS CONTROLÁVEIS: UMA ABORDAGEM DE SISTEMAS DINÁMICOS \\ PARA A TEORIA DO CONTROLE
}

\author{
Roberto Cáceres Anco
}

Este exemplar corresponde à redação final da dissertação apresentada por Roberto Cáceres Anco, devidamente corrigida e aprovada pela Comissão Julgadora

São Paulo, 20 de dezembro de 1996

\section{Comissão Julgadora}

- Prof. Dr. Pedro Aladar Tonelli - IME-USP

- Prof. Dr. Luciano Barbanti - IME-USP

- Prof. Dr. Luiz Antonio B. San Martin - IMECC-UNICAMP 


\section{RESUMO}

Sistemas de controle da forma $\dot{x}=X_{0}(x)+\sum_{i=1}^{m} u_{i}(t) X_{i}(x)$. numa variedade $M \subset \mathbb{R}^{n}$ podem ser considerados como sistemas dinâmicos num espaço métrico $\mathcal{U} \times M$, onde $\mathcal{U}$ é um conjunto de funções de controle admissível, que tomam valores numa faixa de controle compacto, conexo $U \subset \mathbb{R}^{m}$. Em particular mostramos a relação entre caos topológico e controlabilidade em regiões menores do espaço estado $M$ (conjuntos controláveis) em sistemas de controle não linear do tipo acima, contínuos e discretos. Este trabalho sistematiza numa forma unificada uma série de resultados devido a Kliemann-Colonius e Albertini-Sontag.

\section{ABSTRACT}

Associated to control systems of type $\dot{x}=X_{0}(x)+\sum_{i=1}^{m} u_{i}(t) X_{i}(x)$ Kliemann and Colonius construct a dynamical system, whose properties related to topological chaos are the main subject of this dissertation. An unified treatment of discrete and continuous cases are discussed. 
À memória de meu pai, Juan.

Àqueles que fizeram com que eu chegasse até aqui. 


\section{Conteúdo}

Introdução $\quad$ iv

1 Resultados preliminares de análise e dinâmica topológica 1

1.1 Ações de um grupo topológico num espaço topológico . ..................

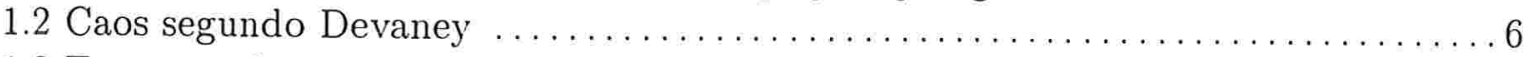

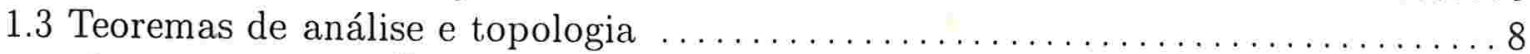

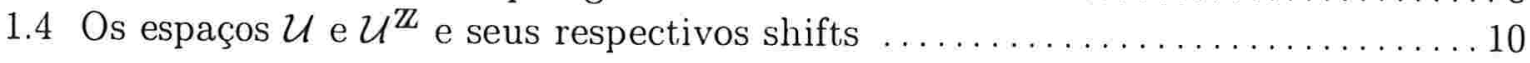

2 Teoria de conjuntos controláveis 24

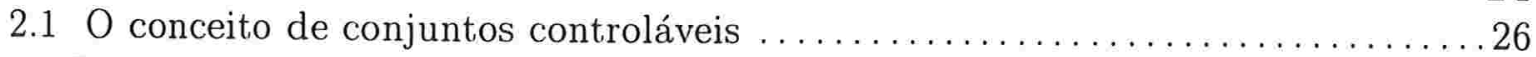

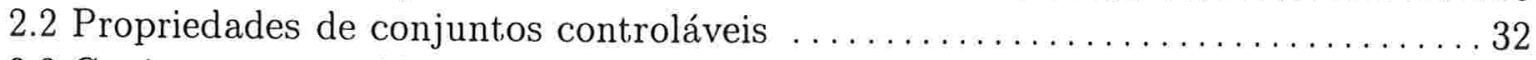

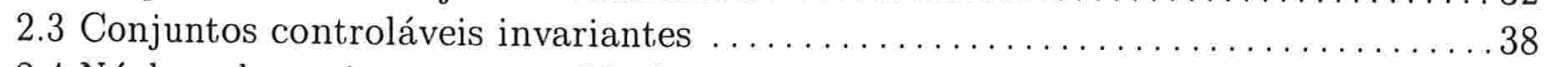

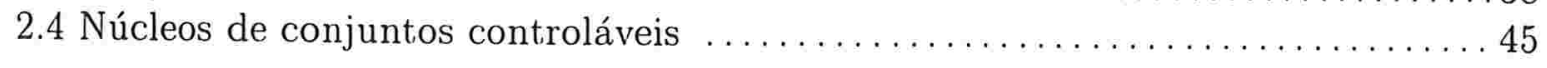

3 O sistema dinâmico associado ao sistema de controle 48

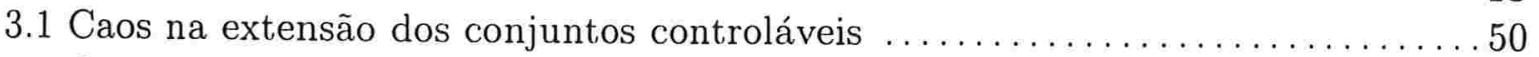

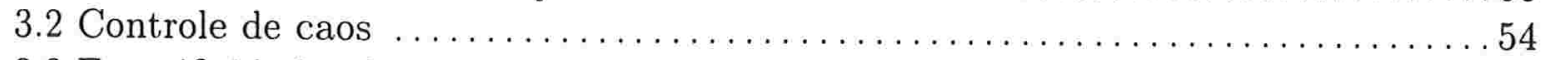

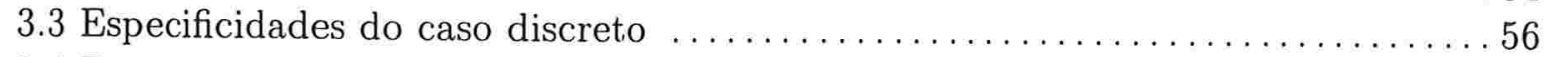

3.4 Decomposição de $\mathcal{U} \times M$ em componentes caóticos $\ldots \ldots \ldots \ldots \ldots \ldots \ldots \ldots 6$

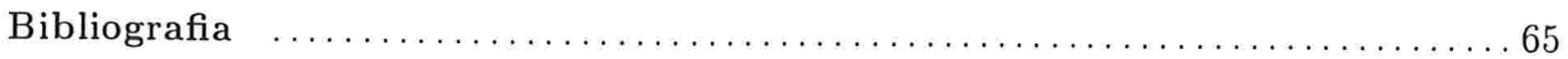




\section{Introdução}

A descrição quantitativa do mundo real depende de uma relação delicada de fenômenos contímuos e discretos, cuja compreensão ajudará ao homem explicar-se cientificamente a realidade que o circunda. Na verdade os fenômenos discretos são os primeiros a serem notados. Como proclamou P. Montel, "funções, da mesma forma que seres vivos, são caracterizados por suas singularidades".

No estudo de sistemas de controle não linear as questões de controlabilidade são caracterizadas por seus aspectos de caráter local e global do sistema. Ao contrário do que acontece no caso linear, onde a controlabilidade é uma propriedade global no espaço estado, os sistemas não lineares podem possuir várias regiões no espaço estado no qual a propriedade de controlabilidade local é válida sem serem globalmente controláveis, são estas regiões que tentaremos caracterizar. É, então, um conceito de extrema importância os chamados conjuntos controláveis, que vêm sendo estudados nas últimas décadas. Estes conjuntos são basicamente regiões no espaço estado, onde, partindo de um ponto, sempre é possível atingir pelo menos uma vizinhança de um ponto próximo.

O objetivo deste trabalho é estabelecer de forma acessivel a relação entre caos topológico e controlabilidade em regiões menores de um espaço estado (conjuntos controláveis) em sistemas de controle não linear contínuos e discretos.

Nosso trabalho baseia-se nos artigos [4], [1] de Fritz Colonius-Wolfgang Kliemann e Fransesca Albertini-Eduardo D. Sontag, respectivamente. Em particular, foi Kliemann que introduziu este conceito na década de oitenta, provando que em dimensão um estes 
conjuntos são simplesmente as singularidades ou pontos críticos de uma função $f_{u}(x)$ para "l variando num conjunto de entradas.

O interesse atual e as idéias da teoria apresentada está em uma gama de aplicações: na teoria de sistemas de controle mesmo [5], em sistemas estocásticos [12], na teoria de semigrupos [15], nà teoria de perturbações de uma equação diferencial [6] e outras áreas da ciência, em particular em sistemas dinâmicos caóticos. Estes últimos têm ocupado a atenção de inúmeros matemáticos como Poincaré, Andronov, Pontriaguin, Anosov, Smale, Palis, entre outros, que atualmente têm um desenvolvimento marcante.

A estrutura deste trabalho é de três capítulos. No primeiro capítulo damos uma informação das noções básicas de dinâmica topológica e suas propriedades, definimos o caos segundo Devaney [8] e comentamos suas propriedades. Também introduzimos os resultados de análise que são necessários na dissertação. Acreditamos que neste capítulo o mais importante são os exemplos de sistemas dinâmicos caóticos, tanto num espaço topológico abstrato - o espaço shift - como em variedades diferenciáveis.

O capítulo dois introduz a noção de conjuntos controláveis numa forma unificada e desenvolve os rudimentos das propriedades de conjuntos controláveis; são discutidos também conjuntos controláveis invariantes. Em particular, são provadas muitas propriedades destes últimos. Cabe ressaltar que o núcleo de um conjunto controlável é um conceito que substitui o interior de conjuntos controláveis, principalmente no caso discreto. A razão é que certas propriedades não são necessariamente válidas no interior de conjuntos controláveis.

Para concluir, o capítulo três estuda a relação entre os sistemas de controle contínuo e discreto, e um sistema dinâmico cruzado (skew product flow) originado a partir destes sistemas de controle, eles são caóticos na extensão de um conjunto controlável. Observe que eles não provêm de uma equação diferencial. Também é demonstrado um teorema importante que sob condições de acessibilidade local, para cada sistema cruzado caótico 
existe um conjunto controlável com interior não vazio e reciprocamente. Um exemplo mostra as especificidades do caso discreto.

A última seção trata da decomposição do espaço estado em componentes caóticos. mais ou menos em analogia à decomposição espectral de conjuntos básicos de Smale; antes são apresentadas uma discussão dos sistemas Axioma $\mathrm{A}$ e a decomposição espectral.

Em resumo, o que pretendemos fazer é mostrar a relação de caos e controlabilidade e suas interrelações, ilustrando tais fenômenos com exemplos geométricos.

Finalmente, desejamos expressar nossos agradecimentos ao Prof. Dr. Pedro Aladar Tonelli, por sua orientação, sugestões, comentários e seu espírito crítico no decorrer deste trabalho, e ao CNPq, pela subvenção.

Aproveito também a oportunidade para agradecer à Profa. Dra. Élvia Mureb Sallum, cuja clareza e energia em suas lições dadas no curso de Cálculo Avançado aqui no IME, muito influíram em nossa formação e por introduzir-nos na teoria de sistemas dinâmicos. 


\section{Capítulo 1}

\section{Resultados preliminares de análise e dinâmica topológica}

Neste primeiro capítulo estabelecemos os resultados e definições mais importantes da teoria qualitativa da dinâmica topológica. Apresentamos também resultados de análise que serão necessários ao trabalho e discutimos ainda o "shift" contínuo e discreto no espaço das funções mensuráveis e provamos o comportamento caótico de sua dinâmica. As definições e demonstrações dos fatos aqui mencionados podem ser encontrados em [3], [4], [8] e [9]. Terminamos o capítulo estabelecendo as propriedades da aplicação "shift" e dando exemplos de caos em sistemas dinâmicos diferenciáveis.

\subsection{Ações de um grupo topológico num espaço to- pológico}

Aqui introduzimos a noção de ação de grupo topológico $G$ em um espaço topológico abstrato. Uma ação contínua de um grupo $G$ num espaço topológico $D$ é uma aplicação $\Phi: G \times D \rightarrow D$ contínua tal que ${ }^{1}$ :

a1) $\Phi(e, x)=x, \forall x \in D$, onde $e$ é a identidade de $G$;

a2) $\Phi(t . s, x)=\Phi(t, \Phi(s, x)), \forall t, s \in G$ e $x \in D$.

\footnotetext{
${ }^{1}$ Neste trabalho a ação será uma ação à esquerda.
} 
Denotamos por $\Phi_{t}: D \rightarrow D$ a aplicação contínua $\Phi_{t}(x)=\Phi(t, x), \forall t \in G$. Então $\Phi_{t}$ é um homeomorfismo de $D$ sobre $D$ : a inversa de $\Phi_{t}$ é justamente $\Phi_{-t}$.

Nós nos ocuparemos por enquanto só com dois grupos, $G=\mathbb{R}$ e $\mathbb{Z}$. Este é o caso de tempo contínuo e discreto, respectivamente. Ainda vários exemplos são dados em sistemas dinâmicos diferenciáveis. Nesse caso, o espaço estado será uma variedade suave.

Um sistema dinâmico ou fluxo contínuo (discreto) topológico é definido como uma ação de um grupo $G$ em um espaço topológico. Podemos ainda denotar por $(D, G, \Phi)$, onde

- $D$ - espaço topológico, chamado espaço estado;

- $G$ - conjunto tempo;

- $\Phi$ - operador evolução.

\section{Exemplos}

1. Seja $D$ um espaço topológico, $G$ o grupo dos homeomorfismos de $D$ sobre $D$. $G^{\prime}$ um subgrupo de $G$, então a $(f, x) \rightarrow f(x)\left(f \in G^{\prime}, x \in D\right)$ de $G^{\prime} \times D$ em $D$ define uma ação dos homeomorfismos.

2. Seja $\varphi \in G$. Então a aplicação $(x, n) \rightarrow \varphi^{n}(x)$ define uma ação dos inteiros $\mathbb{Z}$ em D.

Um ponto $x$ em $D$ é dito fixo para o fluxo $\Phi$ se $\Phi(t, x)=x$ para todo $t$. A função de $t, \Phi(t, x)$ é dita o movimento por $x$. Ainda dizemos que $x$ é um ponto periódico e o movimento $\Phi(t, x)$ é dito periódico se existe $\tau>0$ tal que $\Phi(\tau+t, x)=\Phi(t, x), \forall t$. O número $\tau$ é chamado de período. A trajetória de $x \in D$ é o conjunto

$$
\gamma(x)=\{\Phi(t, x): t \in G\}
$$


(isto ć também reforido como órbita por $x$ ). A semitrajotória positiva e a semitrajetória negativa são definiclos por

$$
\hat{\imath}^{+}(x)=\{\Phi(t, x): t \geq 0\} \quad \vartheta^{-} \quad \hat{\gamma}^{-}(x)=\{\Phi(t \ldots x): t \leq 0\} .
$$

respectivamente.

Algumas vezes a órbita de um ponto por ? permanece dentro de uma regiāo particular de espaço de fase para $t \in G$. Um conjunto $\Lambda \subset D$ é dito invariante por $\Phi$ se,$(x) \subset \Lambda$ quando $x \in \Lambda$. ainda o conjunto $\Lambda$ í dito positivamente invariante ou negativamente invariante, se as órbitas de seus elementos permanecem dentro deles para $t \geq 0$ ou $t \leq 0$. isto é. $\gamma^{+}(x) \subset \Lambda$ ou $\gamma^{-}(x) \subset \Lambda$. quando $x \in \Lambda$.

É claro que a órbita de qualquer ponto é um exemplo de um conjunto invariante. logo pontos fixos, ciclos e órbitas fechadas são conjuntos invariantes; mas é necessário salientar que conjuntos invariantes são ditos minimais quando eles são não vazios, fechados e não contêm subconjuntos próprios com estas propriedades.

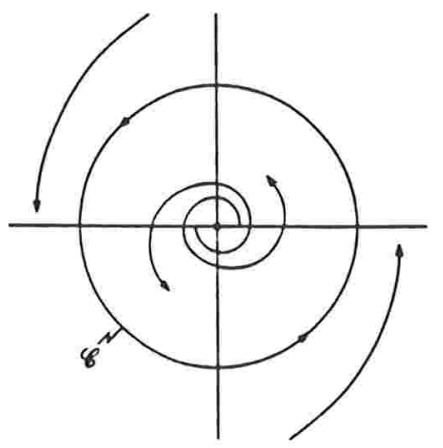

(a)

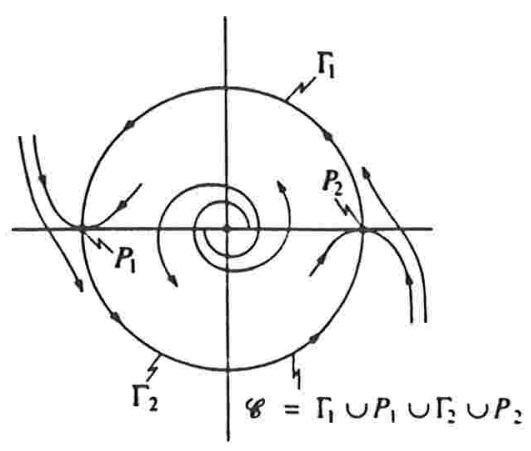

(b)

Figura 1.1

A Figura 1.1 mostra o retrato de fase de dois fluxos em $\mathbb{R}^{2}$, onde o círculo \& é conjunto invariante em ambos os casos. Mas, em (a). $\mathscr{B}$ não tem subconjuntos próprios que são eles mesmos invariantes, isto é. é conjunto minimal. Porém. em (b). $\mathscr{B}$ é a união disjunta dos conjuntos invariantes $P_{1}, P_{2}, \Gamma_{1}, \Gamma_{2}$. 
Formas mais sutis de recorrencia de que periodicidade podem ocorrer em sistemas dinamicos e as seguintes nocoes as descrevem

Lim ponto $x \in D$ i um ponte nà errante para o fluxo $\Phi$ se para toda vizinhança² 1 . de $x$. existe algum $t>t_{0}>0$ para o qual $\Phi_{t}\left(I^{\prime}\right) \cap I^{*} \neq \emptyset$. Caso contrário. dizemos que $x$ é errante. Vide Figura 1.2 .

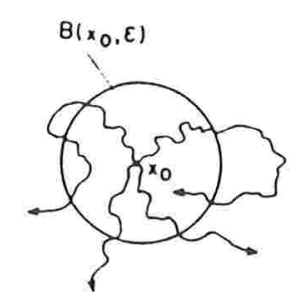

Figura 1.2: Conceitos de recorrência

O conjunto de pontos não errantes para $\Phi$ é chamado conjunto não errante. denotado por $\Omega(\Phi)$. É fácil ver que pontos fixos e órbitas periódicas estão em $\Omega$. Para descrever o comportamento assintótico das órbitas dizemos que se $(D, G . \Phi)$ é um sistema dinâmico contínuo (discreto) e $x \in D$. o conjunto $w$-limite de $x$ é $w(x)=\left\{y \in D: \exists\left\{t_{k}\right\}_{k \in \mathbb{N}} \subset G\right.$. $\lim _{k \rightarrow \infty} t_{k}=\infty$ tais que $\left.y=\lim _{k \rightarrow \infty} \Phi\left(t_{k}, x\right)\right\}$. O conjunto $a$-limite é definido analogamente. quando $k \rightarrow-\infty$.

O conjunto de todos os pontos a e w-limite de $x$ é conhecido como o conjunto a $e$ $w$-limite de $x$, denotados por $L_{a}(x)$ e $L_{w}(x)$. respectivamente. É fácil verificar que estes conjuntos são invariantes pelo fluxo; de fato: seja $z=\Phi_{t}(y), t \in \mathbb{R}$, onde $y$ satisfaz a definição de $w$-limite. então $\lim _{r_{\rightarrow} \infty} \Phi_{t_{i}+t}(x)=z, \log o z$ e $y$ pertencem ao mesmo conjunto limite de $x$.

Um ponto $x \in D$ é dito recorrente se $x \in w(x)$. o conjunto $u^{\prime}$-limite sob $\Phi$. logo $(D, G, \Phi)$ é chamado recorrente. se todo $x \in D$ é recorrente. Seja $\epsilon . T>0$. Lma $(\epsilon . T)$ cadeia de $x \in D$ para $y \in D$ é dado por $n \in \mathbb{N} . x=x_{0} \ldots x_{n}=y$ em $D . t_{0} \ldots \ldots t_{n-1}>T$

\footnotetext{
${ }^{2}$ Ao longo deste trabalho. vizinhança significa vizinh hança aberta
} 
tal que $d\left(\Phi\left(t_{i}, x_{i}\right), x_{i+1}\right)<\epsilon$ para $i=0, \ldots n-1$, onde $d(\cdot, \cdot)$ é a métrica em $D$. O conjunto limite por cadeia de $x \in D$ é dado por $\Gamma(x)=\{y \in D: \forall \epsilon, T>0$ existe uma $(\epsilon, T)$-cadeia de $x$ a $y\}$, e o conjunto recorrente por cadeia é definido como $C R=\{x \in D ; x \in \Gamma(x)\}$. Finalmente, o fluxo $(D, G, \Phi)$ é recorrente por cadeia, se $D=C R$, e ainda transitivo por cadeia, se $y \in \Gamma(x), \forall x, y \in D$.

(Observe que os conjuntos $\alpha$ e $w$-limites são subconjuntos de $\Omega$ para qualquer $x$.)

Para completar esta seção, vamos lembrar outras noções de dinâmica topológica. O sistema dinâmico $\Phi: D \rightarrow D$ é topologicamente transitivo se existe $x \in D$ tal que $w(x)=D$. Esta propriedade qualitativa diz que não é possível decompor o espaço estado em vários subespaços.

Exemplo 1: Consideremos uma equação diferencial no toro $T^{2}=S^{1} \times S^{1}$

$$
\left\{\begin{array}{l}
\dot{\theta}_{1}=w_{1} \\
\dot{\theta}_{2}=w_{2}
\end{array} \quad\left(\theta_{1}, \theta_{2}\right) \in T^{2},\right.
$$

com $\frac{w_{1}}{w_{2}}=$ irracional.

O fluxo gerado por esta equação é topologicamente transitivo. Todas as evoluções deste sistema são quasi-periódicas no sentido que vários movimentos periódicos diferentes, com freqüências independentes, se combinam (a freqüência de um movimento periódico é o número de períodos por segundo; períodos longos correspondem portanto a baixas freqüências, períodos curtos a altas freqüências) cada curva evolução é densa no toro, isto é, $w(x)=T^{2}$.

Dizemos que o sistema dinâmico $\Phi: D \rightarrow D$ é topologicamente mixing se para quaisquer conjuntos abertos $A_{1}, A_{2}$ não vazios de $D$, existe $t>0, t_{0}, t \in G$, tal que $A_{1} \cap \Phi\left(-n t_{0}+t_{1} ; A_{2}\right) \neq \emptyset, \forall n \in \mathbb{N}$. Isto também poderia ser entendido assim: se $A_{1}$ é qualquer conjunto aberto no domínio da função, então algum ponto $x$ de $A_{1}$ eventualmente chegará em toda vizinhança de cada ponto do domínio sob iteração da função. 
Algumas vezes esta definição é utilizada para definir a transitividade topológica. na verdade topologicamente mixing implica transitividade topológica quando o espaço estado é compacto, vide R. Mañe [11]. Tal fato será usado mais tarde.

Seja $D$ um espaço métrico com a métrica $d$. Dizemos que o sistema dinâmico đ : $D \rightarrow D$ exibe dependência sensitiva das condiçôes iniciais se existe uma constante de sensitividade $\delta>0$ tal que para todo $x \in D$ e $\forall$ vizinhança $\mathcal{N}$ de $x$, existem $y$ em $\mathcal{N}_{x}$ e um tempo $t>0$ tais que $d(\Phi(t, x), \Phi(t, y)) \geq \delta$. Em outras palavras, podemos dizer que $\Phi$ é sensitivo quando existe uma constante positiva $\delta>0$ tal que para quaisquer $q \in w(x)$ e quaisquer $\epsilon>0$ existem $t_{1}, t_{2}, t>0$ tal que $d\left(\Phi\left(t_{1}, x\right), q\right)<\epsilon, d\left(\Phi\left(t_{2}, x\right), q\right)<\epsilon$. Mas $d\left(\Phi\left(t_{1}+t, x\right), \Phi\left(t_{2}+t, x\right)\right)>\delta$.

Entendemos esta propriedade métrica como a imprevisibilidade do tempo da dinâmica no domínio da função. Agora vamos dar um exemplo de sensitividade em condições iniciais.

Exemplo 2: Seja a seguinte equação diferencial em $\mathbb{R}$

$$
\dot{x}=\frac{\mathrm{d} x}{\mathrm{~d} t}=a x, \quad x \in \mathbb{R}
$$

onde $a>0$.

O fluxo gerado por esta equação é dado por $\Phi(t, x)=e^{a t} x$ tem dependência sensitiva em condições iniciais em $\mathbb{R}$, pois para qualquer $x_{1}, x_{2} \in \mathbb{R}$, com $x_{1} \neq x_{2}, d\left(\Phi\left(t, x_{1}\right), \Phi\left(t, x_{2}\right)\right)$ $=e^{a t} d\left(x_{1}, x_{2}\right)>2^{t} \epsilon$. Portanto, a distância da imagem de dois pontos próximos cresce exponencialmente no tempo.

\subsection{Caos segundo Devaney}

Como em todo fenômeno natural, social e do pensamento humano, o caos e ordem são aspectos de um mesmo processo, onde o novo se impõe sob o velho. 
Nesta seção vamos fazer alguns comentários sobre o conceito desse fenômeno chamado caos. cuja definição matemática ainda não é de aceitação universal, pela existência de muitas possíveis definições, umas fracas e outras fortes. Entre elas poderíamos assinalar os conceitos métricos, que usam teoria da medida e outros de caráter topológico. Adotamos uma definição de caus topológico dada por Devaney [8] que destaca três propriedades fundamentais de caos.

Definição 1.2.1 (Devaney) Seja D um espaço métrico. Uma aplicação contínua $\Phi: D \rightarrow D$ diz-se caótica em $D$ se satisfaz às seguintes condições:

(Ca1) os pontos periódicos de $\Phi$ são densos em $D$;

(Ca2) $\Phi$ é topologicamente transitivo;

(Ca3) $\Phi$ tem dependência sensitiva em condições iniciais.

Em resumo, esta definição diz que uma aplicação caótica observa uma certa quantidade de regularidade, que é evidenciada pelo fato que sempre podemos achar uma órbita periódica em cada vizinhança de cada ponto no domínio $D$ da aplicação, por menor que seja a vizinhança.

Por outro lado, o espaço estado $D$ é indecomponível por causa da transitividade topológica no sentido que para cada $x, y \in D$ e $\epsilon>0$, existem $x=x_{0}, x_{1}, \ldots, x_{n-1}, x_{n}=y$ e $t_{1}, \ldots, t_{n} \geq 1$ tais que a distância de $\Phi_{t_{i}}\left(x_{i-1}\right)$ a $x_{i}$ é menor que $\epsilon$. Poderíamos também entender isto como que o domínio é bem alterado pela função, pois se escolhemos qualquer conjunto aberto, então podemos achar um ponto em cada outro conjunto aberto, a que eventualmente terminará no primeiro conjunto sob iteração da aplicação. Além disso, dependência sensitiva das condições iniciais diz que se estamos usando uma função iterada para modelar o comportamento assintótico, tais como crescimento populacional, o tempo ou desempenho econômico, a menor mudança na posição inicial implica em resultados 
dramaticamente diferentes entre o comportamento predito e o comportamento atual do sistema que estamos modelando, isto chama-se imprevisibilidade.

Agora, observemos os exemplos da seção anterior. No primeiro exemplo, o sistema dinâmico é definido num espaço topológico compacto, invariante, onde não há órbitas periódicas nem dependência sensitiva em condições iniciais. No seguindo exemplo $\Phi(t, x)$ é topologicamente transitivo nos conjuntos não compactos $(0, \infty)$ e $(-\infty, 0)$, porém não tem órbitas periódicas.

\subsection{Teoremas de análise e topologia}

Nesta seção vamos comentar os resultados de análise e topologia que usaremos, principalmente na seção seguinte. As demonstrações dos fatos aqui enunciados podem ser encontrados em H. Brezis [3] e R. Engelking [9].

Teorema 1.3.1 Seja $\varphi \in\left[L^{1}\left(\mathbb{R}, \mathbb{R}^{m}\right)\right]^{*}$. Então existe um único $\psi \in L^{\infty}\left(\mathbb{R}, \mathbb{R}^{m}\right)$ tal que

$$
\langle\varphi, f\rangle=\int \psi f, \quad \forall f \in L^{1}\left(\mathbb{R}, \mathbb{R}^{m}\right) .
$$

Além disso,

$$
\|\psi\|_{L^{\infty}}=\|\varphi\|_{\left(L^{1}\right)^{*}} .
$$

Demonstração: Vide [3], p.63.

O teorema afirma que toda forma linear e contínua sobre $L^{1}$ é representada por meio de uma função de $L^{\infty}$. A aplicação $\varphi \rightarrow \psi$ é uma isometria que permite identificar $\left(L^{1}\right)^{*}=L^{\infty}$.

Lembramos também que o espaço $L^{p}\left(\mathbb{R}, \mathbb{R}^{m}\right)$ é separável para $1 \leq p \leq+\infty$. Quando uma topologia possui menos abertos, também possui mais compactos. Nesse contexto, é importante a seguinte noção: sejam $E, E^{*}$ o espaço completo e o seu dual, respectivamente. A topologia $w^{*}$ é a topologia menos fina sob $E^{*}$ que faz contínua a todas as aplicações $f_{x}: E^{*} \rightarrow \mathbb{R}$ definido por $f_{x}(\varphi)=\langle\varphi, x\rangle, \forall x \in E$. 
Topologia $w^{*}$ significará topologia fraca estrela: a estrela lembra que trabalhamos sobre o dual, designado por $E^{*}$. Como a topologia $u^{*}$ é separável, então para $k \in \mathbb{N}$. $f_{1}, \ldots, f_{k} \in E, \varphi_{j} \in E^{*}$ e $\epsilon>0$, a família

$$
\mathcal{N}_{\epsilon}\left(f_{1}, \ldots, f_{k}\right)=\left\{\varphi \in E^{*}:\left|\left\langle\varphi-\varphi_{j} ; f_{j}\right\rangle\right|<\epsilon, \forall j=1, \ldots, k\right\}
$$

forma uma base da topologia $w^{*}$ de $E^{*}$.

O resultado seguinte é devido a Banach-Alaoglu.

Teorema 1.3.2 Seja E um espaço de Banach. Então $B_{1}^{*}=\left\{x^{*} \in E^{*} ;\left\|x^{*}\right\| \leq 1\right\}$ é compacto na topologia $w^{*}$.

Demonstração: Vide [3], p.42.

Num curso normal de análise funcional demonstra-se um resultado que diz que a bola unitária fechada de um espaço normado de dimensão infinita nunca é compacta na topologia forte, se compreende agora a importância fundamental da topologia $w^{*} \mathrm{e}$ do fato anterior.

Teorema 1.3.3 Se E é um espaço de Banach, então a topologia $w^{*}$ em $B_{1}^{*}$ é metrizável se e somente se E é separável.

Demonstração: Vide [9], p.48.

A primeira parte quer dizer que existe uma métrica definida sobre $B_{1}^{*}$ tal que a topologia associada coincide com a topologia $w^{*}$ sobre $B_{1}^{*}$.

É conhecido que todo espaço métrico num espaço compacto é completo.

Teorema 1.3.4 Todo espaço métrico num espaço compacto é totalmente limitado.

Demonstração: Vide [8], p.275. 
Teorema 1.3.5 Um espaço métrico $(X, d)$ possui uma métrica d' equivalente. totalmente limitada se e somente se $X$ é um espaço separável.

Demonstração: Vide [8], p.268.

\subsection{Os espaços $\mathcal{U}$ e $U^{\mathbb{Z}}$ e seus respectivos shifts}

Agora discutimos um exemplo particular de dinâmica topológica, que aparecerá na teoria de controle.

Seja $\mathcal{U}$ o conjunto $\left(u_{1}(t), \ldots, u_{n}(t)\right)=: u(t) \in \mathcal{U}=\left\{u: \mathbb{R} \rightarrow U \subset \mathbb{R}^{m \geq 1}\right.$ tal que $u$ é localmente integrável $\}$, onde $U \subset \mathbb{R}^{m \geq 1}$ é compacto, convexo e conexo, que contém pelo menos dois pontos diferentes. No caso discreto, $U^{\mathbb{Z}}=\{u: \mathbb{Z} \rightarrow U\}$ e int $U$ conexo, isto é, $U \subseteq \overline{\operatorname{int} U}^{3}$.

Definição 1.4.1 Consideremos $\mathcal{U}$ um dos conjuntos de funções definidos acima. A aplicação $h: \mathbb{R} \times \mathcal{U} \rightarrow \mathcal{U}$ definida por $h(t, u(\cdot))=u(\cdot+t)$ será chamada o shift à direita por $t \in G$ para $h(t, \cdot): \mathcal{U} \rightarrow \mathcal{U}$, depois usaremos simplesmente $h_{t}$.

$\langle\cdot, \cdot\rangle$ denota o produto interior em $\mathbb{R}^{m}$, em todo trabalho.

\section{Proposição 1.4.1 Consideremos}

- d a métrica em $\mathcal{U}$ definida por

$$
d(u, v)=\sum_{n=1}^{\infty} 2^{-n} \frac{\left|\int_{\mathbb{R}}\left\langle u(t)-v(t) ; f_{n}(t)\right\rangle \mathrm{d} t\right|}{1+\left|\int_{\mathbb{R}}\left\langle u(t)-v(t), f_{n}(t)\right\rangle \mathrm{d} t\right|}
$$

- $\left\{f_{n}: n \in \mathbb{N}\right\} \subset L^{1}\left(\mathbb{R}, \mathbb{R}^{m}\right)$ conjunto denso e enumerável;

Se o conjunto $\mathcal{U}$ é compacto e metrizável na topologia $w^{*}$ de $L^{\infty}\left(\mathbb{R}, \mathbb{R}^{m}\right)=\left[L^{1}\left(\mathbb{R}, \mathbb{R}^{m}\right)\right]^{*}$, então $(\mathcal{U}, d)$ é um espaço métrico compacto, completo e separável.

\footnotetext{
${ }^{3}$ Ao longo deste trabalho int significa interior de um conjunto usual e ${ }^{-}$é o fecho usual.
} 
Demonstração: Pelo Teorema 1.3.1 o espaço dual de $L^{1}\left(\mathbb{R}, \mathbb{R}^{m}\right)$ é $L^{\infty}\left(\mathbb{R} . \mathbb{R}^{m}\right)$. além disso o espaço $L^{1}\left(\mathbb{R}, \mathbb{R}^{m}\right)$ é separável. Agora. utilizando o Teorema 1.3 .3 o espaço dual de um espaço de Banach separável tem uma bola unitária compacta metrizável na topologia $w^{*}$ e a métrica é dada por (1.1). Agora, $\mathcal{U} \subset L^{\infty}\left(\mathbb{R}, \mathbb{R}^{m}\right)$ é limitado na topologia da norma (pois $U$ é compacto) e fechado na topologia $w^{*}$, porque se $u_{n} \in \mathcal{U}, u_{n} \stackrel{w^{*}}{\longrightarrow} u_{0}$, então $\int_{K}\left|u_{0}\right| \mathrm{d} t \leq \sup \left|u_{0}\right| m(K)<\sup \left|u_{0}\right||b-a|<\infty ;$ assim $u_{0}$ é localmente integrável, $u_{0} \in \mathcal{U}$, logo pelo Teorema Banach-Alaoglu's, $\mathcal{U}$ é $w^{*}$-compacto, então $\mathcal{U}$ é um espaço métrico compacto. Portanto $\mathcal{U}$ é completo e finalmente o Teorema 1.3 .5 garante que $\mathcal{U}$ é separável.

No caso discreto, $U^{\mathbb{Z}}$ é compacto metrizável na topologia da convergência pontual, então $\left(U^{\mathbb{Z}}, d\right)$ tem as mesmas propriedades mostradas para o caso contínuo, onde $d$ é dado por

$$
d(u, v)=\sum_{t \in \mathbb{Z}} 2^{-|t|}|u(t)-v(t)|
$$

isto é, $U^{\mathbb{Z}}$ é um espaço métrico compacto, completo e separável.

De agora em diante $\mathcal{U}\left(U^{\mathbb{Z}}\right)^{4}$ será espaço métrico munido com a métrica dada por (1.1) e $\left(1.1^{*}\right)$

Lema 1.4.2 As funções periódicas são densas em $\mathcal{U}$.

Demonstração: Vamos escolher um controle $u^{0} \in \mathcal{U}$ e seja $\mathcal{N}_{u^{0}}$ uma vizinhança de $u^{0}$. Então, para $k \in \mathbb{N}$, existem $\epsilon>0$ e $f_{1}, \ldots, f_{k} \in L^{1}\left(\mathbb{R}, \mathbb{R}^{m}\right)$ tais que

$$
\left\{\begin{array}{l}
u \in L^{\infty}\left(\mathbb{R}, \mathbb{R}^{m}\right) ; u(t) \in U \text { quase sempre e } \\
\left|\int_{\mathbb{R}}\left\langle u^{0}(t)-u(t) ; f_{j}(t)\right\rangle \mathrm{d} t\right|<\epsilon \text { para } j=1, \ldots, k
\end{array}\right\} \subset \mathcal{N}_{u^{0}}
$$

onde este conjunto forma uma base da topologia $w^{*}$ de $\mathcal{U}$. Como $f_{j} \in L^{1}\left(\mathbb{R}, \mathbb{R}^{m}\right)$, então existem $T>0$ e $\epsilon_{0}=\frac{\epsilon}{\operatorname{diam} U}>0$, onde $\operatorname{diam} U=\sup \left\{\left|u_{1}-u_{2}\right|, u_{1}, u_{2} \in U\right\}<\infty$ tais que $\int_{\mathbb{R} \backslash[-T, T]}\left|f_{j}(t)\right|<\epsilon_{0}, \forall j=1, \ldots, k$.

\footnotetext{
${ }^{4}$ Além disso $\mathcal{U}$ denotará ambos os espaços em todo o trabalho.
} 
Agora definamos $u_{p}:[-T . T] \subset \mathbb{R} \rightarrow U$ tais que $u_{p}(t)=u^{0}(t)$ para $t \in[-T . T] \mathrm{e}$ estendemos $u_{p} 2 T$-periodicamente para $\mathbb{R}$. Então $u_{p} \in \mathcal{U}$ e

$$
\begin{aligned}
\left|\int_{\mathbb{R}}\left\langle u^{0}(t)-u_{p}(t) ; f_{j}(t)\right\rangle \mathrm{d} t\right| & =\left|\int_{\mathbb{R} \backslash[-T, T]}\left\langle u^{0}(t)-u(t) ; f_{j}(t)\right\rangle \mathrm{d} t\right| \\
& \leq \operatorname{diam} U \int_{\mathbb{R} \backslash[-T, T]}\left|f_{j}(t)\right| \mathrm{d} t \\
& <\operatorname{diam} U \frac{\epsilon}{\operatorname{diam} U}=\epsilon .
\end{aligned}
$$

Isso mostra que as funções periódicas são densas em $\mathcal{U}$.

OBS: Sabemos que se $u \in \mathcal{U}$ é uma função periódica se e somente se $u$ é um ponto periódico de $(\mathcal{U}, h)$ portanto o Lema 1.4.2 diz que os pontos periódicos são densos em $(\mathcal{U}, h)$. De fato:

$$
\begin{aligned}
u(t+T) & =u(t), \quad \forall t \in \mathbb{R}, \\
\left(h_{T} u\right)(s) & =u(T+s)=u(s), \\
h_{T} u & =u, \forall s \in \mathbb{R} .
\end{aligned}
$$

Agora vamos analisar o espaço shift.

Lema 1.4.3 O shift $h$ define um sistema dinâmico contínuo em $\mathcal{U}$.

\section{Demonstração:}

- Trivialmente $h$ satisfaz as propriedades de grupo, isto é,

$$
\begin{aligned}
& h_{t} \circ h_{s}(u(\cdot))=h_{t}\left(h_{s}(u(\cdot))\right)=h_{t}(u(\cdot+s))=u(\cdot+t+s)=h_{t+s}(u(\cdot)) \\
& h_{0}(u)=u(\cdot+0)=u(\cdot) .
\end{aligned}
$$

- Resta apenas mostrar a continuidade de $h$. Então, sejam uma seqüência $t_{0} \rightarrow t$ em $\mathbb{R}$ e outra $u_{n} \rightarrow u$ em $\mathcal{U}$, logo para todo $f \in L^{1}\left(\mathbb{R}, \mathbb{R}^{m}\right)$, temos

$$
\left|\int_{\mathbb{R}}\left\langle u_{n}\left(t_{n}+\tau\right)-u(t+\tau), f(\tau)\right\rangle \mathrm{d} \tau\right|
$$




$$
\begin{aligned}
= & \left|\int_{\mathbb{R}}\left\langle u_{n}\left(t_{n}+\tau\right)-u_{n}(t+\tau)+u_{n}(t+\tau)-u(t+\tau), f(\tau)\right\rangle \mathrm{d} \tau\right| \\
= & \left|\int_{\mathbb{R}}\left\langle u_{n}\left(t_{n}+\tau\right)-u_{n}(t+\tau), f(\tau)\right\rangle \mathrm{d} \tau+\int_{\mathbb{R}}\left\langle u_{n}(t+\tau)-u(t+\tau), f(\tau)\right\rangle \mathrm{d} \tau\right| \\
\leq & \left|\int_{\mathbb{R}}\left\langle u_{n}\left(t_{n}+\tau\right)-u_{n}(t+\tau), f(\tau)\right\rangle \mathrm{d} \tau\right|+\left|\int_{\mathbb{R}}\left\langle u_{n}(t+\tau)-u(t+\tau), f(\tau)\right\rangle \mathrm{d} \tau\right| \\
= & \left|\int_{\mathbb{R}}\left\langle u_{n}(\tau), f\left(\tau-t_{n}\right)\right\rangle \mathrm{d} \tau-\int_{\mathbb{R}}\left\langle u_{n}(\tau), f(\tau-t)\right\rangle \mathrm{d} \tau\right| \\
& +\left|\int_{\mathbb{R}}\left\langle u_{n}(\tau)-u(\tau), f(\tau-t)\right\rangle \mathrm{d} \tau\right| .
\end{aligned}
$$

É imediato que o segundo somando converge a zero, pois $u_{n} \rightarrow u$ em $\mathcal{U}$. Vamos estimar o primeiro somando:

$$
\left|\int_{\mathbb{R}}\left\langle u_{n}(t) ; f\left(\tau-t_{n}\right)-f(\tau-t)\right\rangle \mathrm{d} \tau\right| \leq \sup _{w \in U}|w| \int_{\mathbb{R}}\left|\left\langle f\left(\tau-t_{n}\right)-f(\tau-t)\right\rangle\right| \mathrm{d} \tau
$$

que converge para zero quando $t_{n} \rightarrow t$ em $\mathbb{R}$.

Portanto, $h\left(t_{n}, u_{n}\right)=u_{n}\left(t_{n}+\cdot\right) \rightarrow u(t+\cdot)$ em $\mathcal{U}$.

O resultado seguinte mostrará a propriedade topológica de nosso espaço shift.

Lema 1.4.4 O sistema dinâmico $(\mathcal{U}, G, h)$ é topologicamente mixing e topologicamente transitivo.

Demonstração: Lembremos que para funções em espaços compactos é imediato que topologicamente mixing implica topologicamente transitivo, logo, como $\mathcal{U}$ é compacto, então bastará provar que $h$ é mixing.

Para $k_{j} \in \mathbb{N}$, existem $\epsilon>0$ e $g_{i j} \in L^{1}\left(\mathbb{R}, \mathbb{R}^{m}\right)$ com $i=1, \ldots, k_{j}, j=1,2$, tais que podemos considerar uma base da topologia $w^{*}$ em $\mathcal{U}$ da seguinte maneira:

$$
\mathcal{V}_{j}=\left\{v \in \mathcal{U},\left|\int_{\mathbb{R}}\left\langle u_{j}(\tau)-v(\tau), g_{i j}(\tau)\right\rangle \mathrm{d} \tau\right|<\epsilon, i=1, \ldots, k_{j}\right\}
$$

onde $u_{j} \in \mathcal{U}$. Então existem $T>0$ e $\epsilon_{0}=\frac{\epsilon}{\operatorname{diam} U}>0$ tais que $\int_{\mathbb{R} \backslash[-T, T]}\left|g_{i j}(\tau)\right| \mathrm{d} \tau<\epsilon_{0}$, $\forall i, j$. Agora, seja $t>2 T$, arbitrário, e vamos definir $v: \mathbb{R} \rightarrow U$ por

$$
v(\tau)= \begin{cases}u_{1}(\tau) & ,-T<\tau<\infty \\ u_{2}(\tau+t) & ,-\infty<\tau \leq-T .\end{cases}
$$


Vamos agora estimar o

$$
\begin{aligned}
\left|\int_{\mathbb{R}}\left\langle u_{1}(\tau)-v(\tau), g_{i 1}(\tau)\right\rangle \mathrm{d} \tau\right| & =\int_{-\infty}^{-T}\left|\left\langle u_{1}(\tau)-v(\tau), g_{i 1}(\tau)\right\rangle\right| \mathrm{d} \tau \\
& \leq \operatorname{diam} U \int_{-\infty}^{-T}\left|g_{i 1}(\tau)\right| \mathrm{d} \tau \\
& <\operatorname{diam} U \frac{\epsilon}{\operatorname{diam} U}=\epsilon, \quad \forall i=1, \ldots, k_{1} .
\end{aligned}
$$

Então $v \in \mathcal{V}_{1}$.

Por outro lado, temos

$$
\begin{aligned}
\int_{\mathbb{R}}\left\langle u_{2}(\tau)-v(\tau-t), g_{i 2}(\tau)\right\rangle \mathrm{d} \tau & =\left|\int_{\mathbb{R}}\left\langle u_{2}(\tau+t)-v(\tau), g_{i 2}(\tau+t)\right\rangle \mathrm{d} \tau\right| \\
& =\left|\int_{-T}^{\infty}\left\langle u_{2}(\tau+t)-u_{1}(\tau) ; g_{i 2}(t+\tau)\right\rangle \mathrm{d} \tau\right| \\
& =\left|\int_{t-T}^{\infty}\left\langle u_{2}(\tau)-u_{1}(\tau-t) ; g_{i 2}(\tau)\right\rangle \mathrm{d} \tau\right| \\
& \leq \operatorname{diam} U \int_{T}^{\infty}\left|g_{i 2}(\tau)\right| \mathrm{d} \tau, \quad \operatorname{pois} t-T \geq T \\
& <\epsilon, \quad \forall i=1, \ldots, k_{2},
\end{aligned}
$$

portanto, $h(-t, v) \in \mathcal{V}_{2}$. Isso mostra que $\mathcal{V}_{1} \cap h\left(-n T_{1}+T_{2}, \mathcal{V}_{2}\right) \neq \emptyset$.

A seguir mostraremos a propriedade métrica do espaço shift.

Lema 1.4.5 O sistema dinâmico $(\mathcal{U}, G, h)$ tem dependência sensitiva às condiçôes iniciais.

Demonstração: Para mostrar a sensitividade devemos exibir $\delta>0$ tal que $\forall u \in \mathcal{U}$ e $\forall \mathcal{N}_{u}, \exists w_{u} \in \mathcal{N}_{u}$ temos que $\mathrm{d}\left(h_{t}(u), h_{t}\left(w_{u}\right)\right) \geq \delta$, para todo $t>0$.

Lembremos que $U \subset \mathbb{R}^{m \geq 1}$ tem ao menos dois pontos e $f_{1}$ de (1.1) é um elemento arbitrário em um subconjunto denso e enumerável de $L^{1}\left(\mathbb{R}, \mathbb{R}^{m}\right)$.

Para qualquer controle $u^{0} \in \mathcal{U}$, existe um controle $v \in \mathcal{U}$ tal que

$$
\int_{\mathbb{R}}\left\langle u^{0}(\tau)-v(\tau), f_{1}(\tau)\right\rangle \mathrm{d} \tau \neq 0
$$


Esta desigualdade é válida para todo $"$ que está em alguma vizinhança de $u^{0}$. logo. como $\mathcal{U}$ é compacto, existe $\delta_{0}>0$ tal que para todo $u \in \mathcal{U}$ existe $v=v(u) \in \mathcal{U}$ tal que

$$
\left|\int_{\mathbb{R}}\left\langle u(\tau)-v(\tau), f_{1}(\tau)\right\rangle \mathrm{d} \tau\right|>\delta_{0}
$$

Agora consideremos $u \in \mathcal{U}$ e uma vizinhança de $n . \mathcal{N}_{u}$, que ainda tomamos da forma

$$
\mathcal{N}_{u}=\left\{w \in \mathcal{U},\left|\int_{\mathbb{R}}\left\langle u(\tau)-w(\tau), g_{i}(\tau)\right\rangle \mathrm{d} \tau\right|<\epsilon, \quad \text { para } i=1, \ldots, k\right\}
$$

Logo existem $T>0$ e $\epsilon_{0}=\frac{\epsilon}{2 \operatorname{diam} U}>0$ tais que $\int_{\mathbb{R} \backslash[-T, T]}\left|g_{i}(\tau)\right| \mathrm{d} \tau<\epsilon_{0}, \forall i=1, \ldots, k$.

Para $u \in \mathcal{U}$ vamos definir $w_{u} \in \mathcal{U}$, da seguinte maneira: $w_{u}: \mathbb{R} \rightarrow U$ tais que

$$
w_{u}(\tau)= \begin{cases}u(\tau) & ,-\infty<\tau<T \\ v_{u}(\tau-T) & , T \leq \tau \leq \infty\end{cases}
$$

onde $v_{u}$ é escolhido de maneira que satisfaz

$$
\left|\int_{\mathbb{R}}\left\langle u(\tau+T)-v_{u}(\tau), f_{1}(\tau)\right\rangle \mathrm{d} \tau\right|>\delta_{0}
$$

Como

$$
\begin{aligned}
\left|\int_{\mathbb{R}}\left\langle u(\tau)-w_{u}(\tau), g_{i}(\tau)\right\rangle \mathrm{d} \tau\right| & =\left|\int_{T}^{\infty}\left\langle u(\tau)-v_{u}(\tau-T), g_{i}(\tau)\right\rangle \mathrm{d} \tau\right| \\
& \leq \operatorname{diam} U \int_{T}^{\infty}\left|g_{i}(\tau)\right| \mathrm{d} \tau \\
& <\epsilon, \quad \forall i=1, \ldots, k .
\end{aligned}
$$

Então $w_{u} \in \mathcal{N}_{u}$. Finalmente,

$$
\begin{aligned}
\mathrm{d}\left(h_{t}(u), h_{t}\left(w_{u}\right)\right) & =\sum_{n=1}^{\infty} 2^{-n} \frac{\left|\int_{\mathbb{R}}\left\langle u(t+\tau)-w_{u}(t+\tau), f_{n}(\tau)\right\rangle \mathrm{d} \tau\right|}{1+\left|\int_{\mathbb{R}}\left\langle u(t+\tau)-w_{u}(t+\tau), f_{n}(\tau)\right\rangle \mathrm{d} \tau\right|} \\
& \geq 2^{-n} \frac{\left|\int_{\mathbb{R}}\left\langle u(t+\tau)-w_{u}(t+\tau), f_{n}(\tau)\right\rangle \mathrm{d} \tau\right|}{1+\left|\int_{\mathbb{R}}\left\langle u(t+\tau)-w_{u}(t+\tau), f_{n}(\tau)\right\rangle \mathrm{d} \tau\right|} \\
& \geq 2^{-1} \delta_{0} \frac{1}{1+\operatorname{diam} U\left\|f_{1}\right\|_{L^{1}\left(\mathbb{R}, \mathbb{R}^{m}\right)}}
\end{aligned}
$$


A última desigualdade decorre da seguinte estimativa. O numerador

$$
\left|\int_{\mathbb{R}}\left\langle u(T+\tau)-w_{u}(T+\tau) \cdot f_{1}(\tau)\right\rangle \mathrm{d} \tau\right|=\left|\int_{\mathbb{R}}\left\langle u(T+\tau)-\iota_{u}(\tau) \cdot f_{1}(\tau)\right\rangle \mathrm{d} \tau\right|>\delta_{0}
$$

por (1.2), e o denominador

$$
\begin{aligned}
& 1+\left|\int_{\mathbb{R}}\left\langle v(T+\tau)-w_{u}(T+\tau) \cdot f_{1}(\tau)\right\rangle \mathrm{d} \tau\right| \leq 1+\operatorname{diam} U \cdot\left\|f_{1}\right\|_{L^{1}\left(\mathbb{R}, \mathbb{R}^{m}\right)} \\
& \frac{1}{1+\left|\int_{\mathbb{R}}\left\langle v(T+\tau)-w_{u}(T+\tau), f_{1}(\tau)\right\rangle \mathrm{d} \tau\right|} \geq \frac{1}{1+\operatorname{diam} U\left\|f_{1}\right\|_{L^{1}\left(\mathbb{R}, \mathbb{R}^{m}\right)}} .
\end{aligned}
$$

Tomemos a constante de sensitividade $\delta=\frac{\delta_{0}}{\left.2(1+\operatorname{diam} U)\left\|f_{1}\right\|_{L^{1}}\right)}$. Portanto $\mathrm{d}\left(h_{t}(u), h_{t}\left(w_{u}\right)\right) \geq \delta$.

Mostramos que o sistema dinâmico shift $(\mathcal{U}, G, h)$ tem um conjunto denso de pontos periódicos, é topologicamente transitivo e tem dependência sensitiva em condições iniciais (de maneira análoga poderíamos mostrar para o caso discreto).

Por conseguinte, o espaço shift é caótico no sentido de Devaney [8], apresentado na Seção 1.2 .

Vamos encerrar este capítulo com dois exemplos de caos em sistemas dinâmicos diferenciáveis. Além das definições dadas para espaços topológicos arbitrários na Seção 1.1, precisamos de algumas definições adicionais que dependem da diferenciabilidade. Assumimos também que o espaço estado $M$ é uma variedade compacta. Consideremos $V \subset N$ uma subvariedade de classe $C^{r}$ e $\Phi: M \rightarrow N$ uma aplicação de classe $C^{k}$, onde $k, r \geq 1$. Dizemos que $\Phi$ é transversal a $V$ num ponto $p \in M$ se $\Phi(p) \notin V$ ou $T N_{\Phi(p)}=T V_{\Phi(p)}+D \Phi_{p}\left(T M_{p}\right)$. Seja $p \in M$ um ponto fixo de $\phi \in \operatorname{Dif}^{r}(M)$. Dizemos que $p$ é um ponto fixo hiperbólico se $D \phi_{p}: T M_{p} \rightarrow T M_{p}$ não tem autovalor de módulo 1 . Isto diz que nenhum do autovalores da matriz $D \phi_{p}$ está no círculo unitário para qualquer $t>0$ fixado, e o conjunto $W^{s}(p)$ dos pontos de $M$ que têm $p$ como $w$-limite denominamos variedade estável de $p$ e o conjunto $W^{u}(p)$ dos pontos que têm $p$ como $\alpha$-limite é chamado variedade instável de $p$. É evidente que estes conjuntos são invariantes. Ainda lembramos 


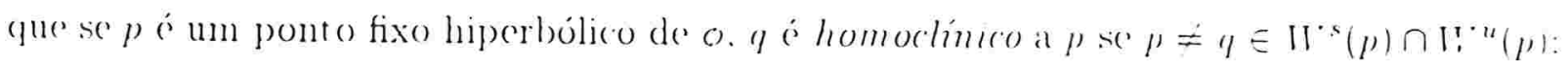

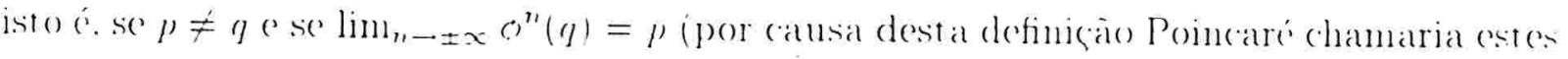
pontos de "biassintóticos").

É preciso notar que esta última definição nos permitirá provar a transitividade topuloggica no segundo exemplo.

Agora formulemos uma nogão de uma classe de sistemas. os assim chamados kupkuSmale. pois o primeiro exemplo de caos na ferradura de Smale é apresentado nesta classe de sistemas.

Sejam $M$ uma rariedade compacta de dimensão $n$ e $\phi \in \operatorname{Dif}^{r}(M I), r \geq 1$. Dizemos que o é um difeomorfismo de Kupka-Smale se:

KS1) todos os pontos periódicos de Ф são hiperbólicos:

KS2) se $x$ e $y$ são pontos periódicos de $\Phi$ então $W^{s}(x)$ é transversal a $W^{* u}(y)$.

Neste caso, dada $\phi \in \operatorname{Dif}^{r}(M I)$, dizemos que $x \in M$ é ponto periódico de $\phi$ se existe $n \in \mathbb{N}^{*} \operatorname{com} \phi^{n}(x)=x$.

OBS: (KS2) significa que os espaços tangentes para as duas subvariedades em qualquer ponto $p$ de interseç̧ão gera todo o espaço tangente da variedade $M$ em $p: T_{p} W^{s}(x)+$ $T_{p} \mathrm{II}^{\cdot u}(y)=T_{p} \Lambda$. Vide Figura 1.3
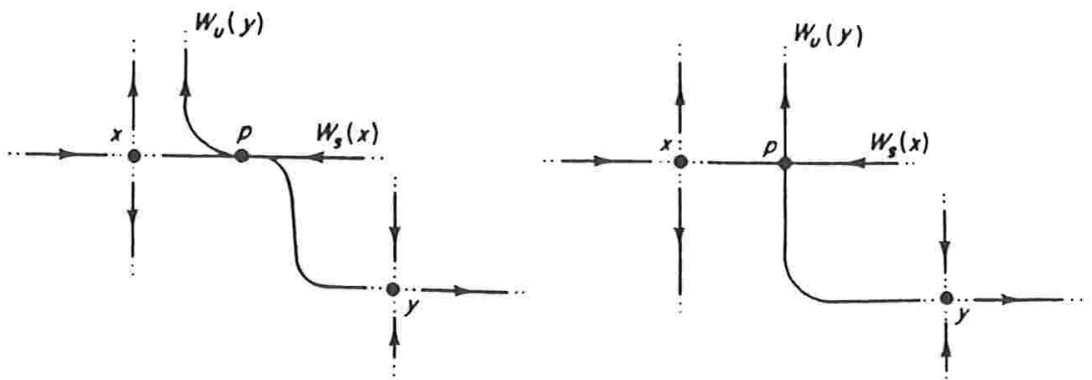

Figura 1.3: Interseç̧ão não transversal em $p$ e interseção transversal em $p$

Nos anos 60. S. Smale examinou um exemplo simples e captou exatamente como 
funciona sua dinámica: ele pegon um quadrado. esticou até torná-lo retangulo longo e

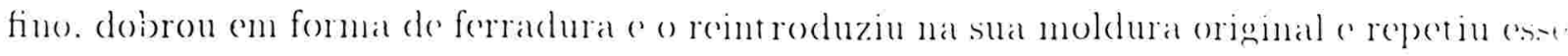
processo indefinidamente, obscrvando que produz uma intrincada estrutura de múltiplas camadas. Isto é chamado a ferradura de Smale. que provaremos que tem sua dinamica ('aótica seģundo Deraney:

Exemplo 3: Sejam $\phi: S^{2} \rightarrow S^{2}$ um difeomorfismo de Kupka-Smale e $\Lambda \subset S^{2} u m$ conjunto não vazio. compacto e invariante, então $\phi: \Lambda \rightarrow \Lambda$ é caótico.

Primeiro vamos construir $\Lambda$. Sejam

$R=\left\{(x, y) \in \mathbb{R}^{2}: x, y \in[0,1]^{2}\right\}$ um quadrado unitário e

$B=\left\{(x, y) \in \mathbb{R}^{2}: x \in[0.1], y \in[0,1 / \mu]\right\}$, onde $\mu \in \mathbb{R}$ é o fator de expansão.

$D=\left\{(x, y) \in \mathbb{R}^{2}: x \in[0,1], y \in[1-1 / \mu, 1]\right\}$ são retângulos horizontais em $R$.
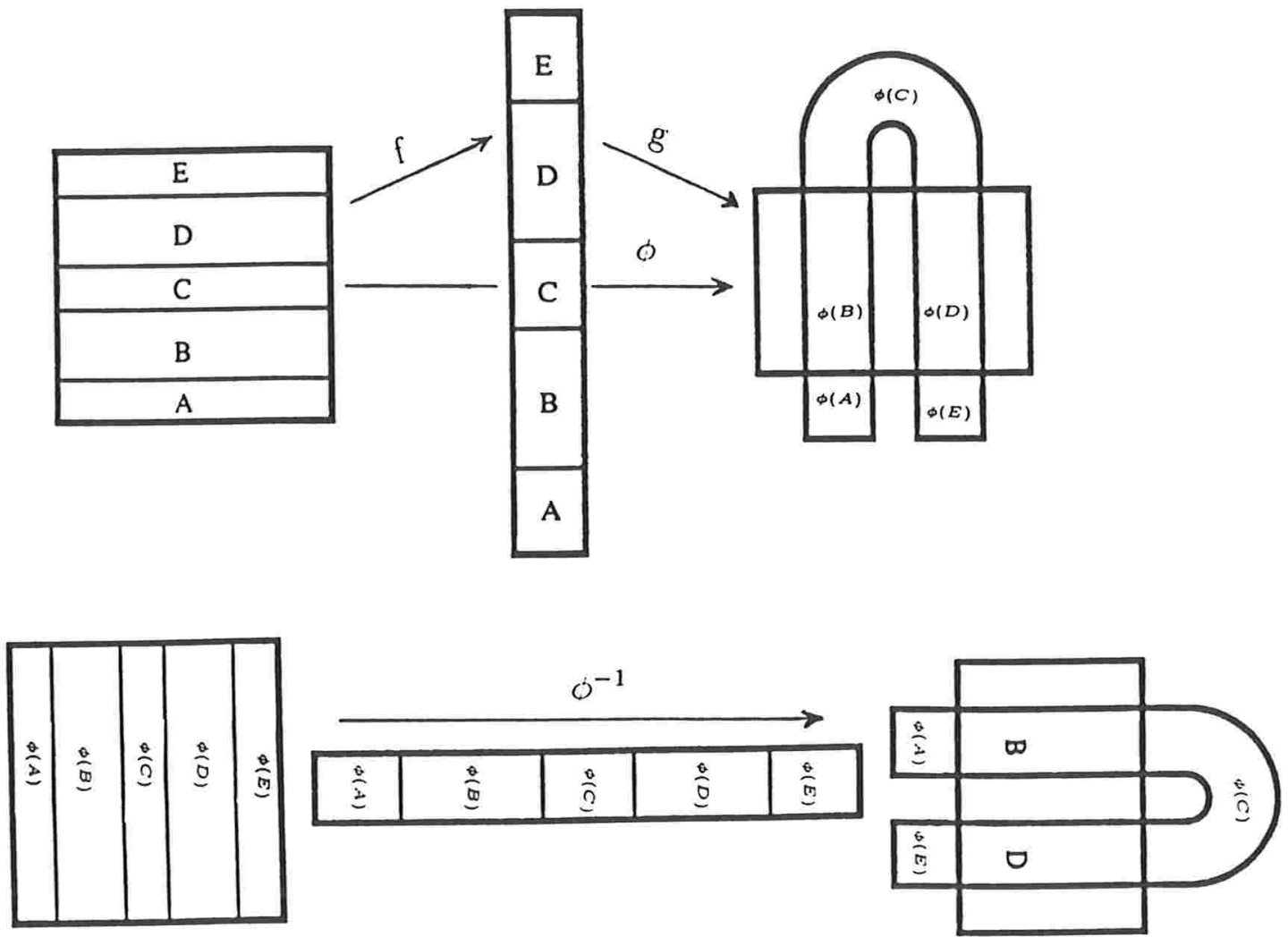

Figura 1.4: A estrutura da aplicação ferradura de Smale 
Além do mais,

$0: B \rightarrow B$ é definido por $\phi(x, y)=\left(\begin{array}{cc}\lambda & 0 \\ 0 & \mu\end{array}\right)\left(\begin{array}{l}x \\ y\end{array}\right) ; \lambda \in\left(0, \frac{1}{2}\right), \mu>2$. onde $\lambda \in \mathbb{R}$ é o fator de contração,

$\phi: D \rightarrow D$ é definido por $\phi(x, y)=\left(\begin{array}{cc}-\lambda & 0 \\ 0 & -\mu\end{array}\right)\left(\begin{array}{l}x \\ y\end{array}\right)+\left(\begin{array}{l}1 \\ \mu\end{array}\right), \lambda \in\left(0, \frac{1}{2}\right), \mu>2$.

Estamos interessados em descrever o conjunto de pontos que sempre fica em $R$ sob iteração de $\phi$ e estes conjuntos de pontos são definidos como:

$$
\begin{aligned}
\Lambda_{v}^{-} & =R \cap \phi(R) \cap \phi^{2}(R) \cap \cdots \cap \phi^{n}(R) \cap \cdots=\bigcap_{n \leq 0}^{-\infty} \phi^{n}(R) \\
\Lambda_{h}^{+} & =R \cap \phi^{-1}(R) \cap \phi^{-2}(R) \cap \cdots \cap \phi^{-n}(R) \cap \cdots=\bigcap_{n \geq 0}^{\infty} \phi^{n}(R) \\
\Lambda & =\Lambda_{h}^{+} \cap \Lambda_{v}^{-}=\bigcap_{n \in \mathbb{Z}} \phi^{n}(R)
\end{aligned}
$$

- $\Lambda$ é um conjunto de Cantor. Como $\Lambda^{+}$e $\Lambda^{-}$são o produto de um intervalo e um conjunto de Cantor linear, portanto $\Lambda$ é um conjunto de Cantor.

- $\Lambda$ é um conjunto invariante. De fato,

$$
\phi\left(\Lambda^{+}\right)=\phi\left(\bigcap_{n \geq 0}^{\infty} \phi^{n}(R)\right)=\bigcap_{n \geq 1}^{\infty} \phi^{n}(R) \subset R,
$$

pois as intersecções estão aninhadas. Portanto $\phi\left(\Lambda^{+}\right)=\Lambda^{+}$. Analogamente, $\phi\left(\Lambda^{-}\right)=$ $\Lambda^{-}$. Logo, $\Lambda$ é invariante.

Agora provaremos as características de caos.

1) Os pontos periódicos de $\phi$ são densos em $\Lambda$. De fato, seja qualquer $x \in \Lambda$ e um quadrado $R_{x}$ contendo $x, \phi^{n}\left(R_{x}\right) \cap R_{x} \neq \emptyset$ para $n$ arbitrariamente grande. A aplicação $\phi^{n}$ linearmente comprime os lados horizontais e expande os lados verticais de $R_{x}$. Da expansão temos que existe um segmento horizontal $I_{h}$ em $R_{x}$ tal que $\phi^{n}\left(I_{h}\right) \subset I_{h}$ e da compressão temos que existe um segmento vertical $I_{v}$ em $R_{x}$ tal que $\phi^{n}\left(I_{v}\right) \supset I_{v}$, logo $I_{h} \cap I_{v}$ é um ponto fixo para $\phi^{n}$. Então $I_{h} \cap I_{v}$ é um ponto periódico para $\phi$. Portanto os pontos periódicos são densos em $\Lambda$. 
2) $\oplus: \Lambda \rightarrow \Lambda$ é topologicamente transitivo. De fato, as variedades estáveis contêm segmentos horizontais e as variedades instáveis contèm segmentos verticais ao longo de todo o quadrado $R$. Logo. as variedades estáveis e instáveis de qualquer órbita periódica tem interseç̧ão não vazia. Portanto. $\Lambda$ é topologicamente transitiva.

3) Sejam $x \in \Lambda$ e $y \in W^{u}(x)$, então para qualquer $n$, tomemos $\delta=2^{n} \epsilon$. Temos $d\left(\phi^{n}(x), \phi^{n}(y)\right) \geq \delta$.

A seguir vamos ver uma definição de outro tipo importante de difeomorfismos que são caóticos, como é mostrado no segundo exemplo.

Seja $M$ uma variedade compacta, dizemos que $\phi \in \operatorname{Dif}^{r}(M), r \geq 1$, é um difeomorfismo de Anosov se:

A1) o fibrado tangente de $M$ se decompõe em uma soma direta contínua $T M=E^{s} \oplus E^{u}$;

A2) $E^{s}$ e $E^{u}$ são invariantes pela derivada de $\phi, D \phi$, isto é, $D \phi_{x} E_{x}^{s}=E_{\phi(x)}^{s}$ e $D \phi_{x} E_{x}^{u}=$ $E_{\phi(x)}^{u}$ para quaisquer $x \in M$;

A3) existem constantes $c>0, c^{\prime}>0$, uma constante $0<\lambda<1$ e uma métrica riemanniana $\|\cdot\|$ sobre $T M$, tais que

$$
\begin{aligned}
& \left\|D \phi^{n}(v)\right\| \leq c \lambda^{n}\|v\| \text { para } v \in E^{s}, \\
& \left\|D \phi^{n}(u)\right\| \geq c^{\prime} \lambda^{-n}\|u\| \text { para } u \in E^{u}{ }^{5} .
\end{aligned}
$$

Como $M$ é compacta, A3) independe da escolha da métrica riemanniana.

\section{OBS:}

- A continuidade da decomposição significa que como $x$ varia em $M$ podemos achar bases variando continuamente para $E_{x}^{s}$ e $E_{x}^{u}$. Em geral, a decomposição não pode ser escolhida suave.

\footnotetext{
${ }^{5} \mathrm{Um}$ conjunto que satisfaz estas três propriedades chama-se hiperbólico.
} 
- As condições de hiperbolicidade de A3) dizem que. infinitesimalmente. vetores em $E^{s}$ $\left(E^{u}\right)$ são contraídos exponencialmente no tempo futuro (passado) em uma proporção exponencial $\lambda$. que é uniforme para todos os pontos de $M$ e todas as escolhas de vetores nos subespaços invariantes. D. Anosov foi o primeiro a fornecer exemplos de sistemas que saisfazem as características anteriores e provar sua estabilidade. Os difeomorfismos de Anosov são considerados com algum detalhe por S. Smale [14].

Exemplo 4: Seja $\phi: T^{2} \rightarrow T^{2}$ um difeomorfismo de Anosov, onde a variedade compacta $C^{\infty}$ é o toro $T^{2}=\mathbb{R}^{2} / \mathbb{Z}^{2}$, induzido pelo isomorfismo linear de $\mathbb{R}^{2}$ dado, em coordenadas canônicas pela matriz $L=\left(\begin{array}{ll}2 & 1 \\ 1 & 1\end{array}\right)$ via a projeção natural $\pi: \mathbb{R}^{2} \rightarrow T^{2}$. então

1) os pontos periódicos de $\phi$ são densos em $T^{2}$;

2) $\phi$ é topologicamente mixing;

3) $\phi$ tem dependência sensitiva em condições iniciais.

De fato:

- Seja um ponto qualquer $p \in T^{2}$ e uma vizinhança $V \subset T^{2}$ tal que $p \in V$, com coordenadas racionais. Vamos assumir que $p$ tem a seguinte forma: $p=\left(\frac{m_{1}}{n}, \frac{m_{2}}{n}\right)$, $m_{1}, m_{2}, n \in \mathbb{Z}$. Tais pontos são claramente densos em $T^{2}$, para ver isso, vamos tomar $n$ suficientemente grande. Afirmamos que $p$ é ponto periódico com período menor ou igual a $n^{2}$. De fato, é claro que existe exatamente $n^{2}$ pontos em $T^{2}$ da forma $\left(\frac{m_{1}}{n}, \frac{m_{2}}{n}\right)$, com $0 \leq m_{1}, m_{2}<n$. Além do mais, como $L$ é uma matriz de coeficientes inteiros, temos que a imagem de qualquer de tais pontos por $\phi$ pode também ser escrita nesta forma. Isto significa que $\phi$ permuta estes pontos, logo existem $i, j \in \mathbb{Z}$ tais que $\phi^{i}(p)=\phi^{j}(p)$ e $|i-j| \leq n^{2}$. Aplicando $\phi^{-i}$ a esta equação, temos $\phi^{-i} \phi^{i}(p)=\phi^{-i+j}(p)$. Então $p=\phi^{j-i}(p)$. Portanto $p$ é ponto periódico em $V$. OBS: A origem de $\mathbb{R}^{2}$ é um ponto fixo hiperbólico. 
- Sejam $A_{1} \cdot A_{2}$ abertos cm $T^{2}$. escolhemos $\leqslant \in A_{1} . \eta E A_{2}$ que sejam homoclinicoa zero. Seja $z>0$. escolhemos um intervalo aberto $I_{*}$ de comprimento $\delta>0$ (m $I^{s}(0)$ contendo $\| 0^{\circ}$ de mancira análoga um intervalo aberto $I_{u} \mathrm{~cm} I^{\cdot u}(0)$ contendo

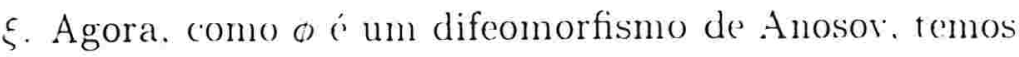

$$
\begin{aligned}
& \left\|D \phi^{\prime \prime}\left(I_{u}\right)\right\| \geq c^{\prime} \lambda^{-n} \text { para } I_{u} \in E^{u} . \\
& \left\|D \phi^{n}\left(I_{s}\right)\right\| \geq c \lambda^{n} \text { para } I_{s} \in E^{s} .
\end{aligned}
$$

Agora escolhemos $n$ suficientemente grande. de maneira que a distância euclideana $d: V_{0} \times V_{0} \rightarrow \mathbb{F}^{+}\left(V_{0}\right.$ é uma vizinhança da origem $)$ satisfaz as seguintes propriedades:
(a) $d\left(\phi^{n}(\xi), 0\right)<\epsilon / 2$,
(b) $d\left(\phi^{-n}(\eta), 0\right)<\epsilon / 2$.
(c) $\left|\lambda_{u}\right|^{n} \delta>\epsilon$.

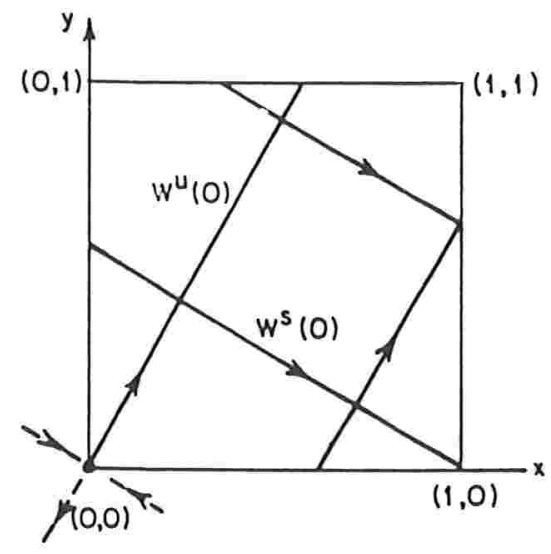

(a)

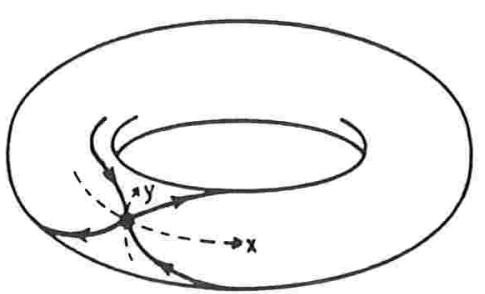

(b)

Figura 1.5: O difeomorfismo de Anosov

(a) no espaço de recobrimento $\mathbb{R}^{2}$ : (b) no $T^{2}$

Como $\phi^{-n}\left(I_{s}\right)$ e $\phi^{n}\left(I_{u}\right)$ são paralelos a $W^{\cdot s}(0)$ e $W^{u}(0)$. respectivamente. disto segue que $\phi^{-n}\left(I_{s}\right) \cap \phi^{n}\left(I_{u}\right) \neq \emptyset$. Vamos supor que $q$ é um ponto desta intersecção. então $p=\phi^{-n}(q) \in A_{1}$ e $\phi^{n}(q) \in A_{2}$. Logo $\phi^{2 n}(p) \in A_{2}$. Portanto. $\Phi: T^{2} \rightarrow T^{2} \dot{\mathrm{e}}$ topologicamente mixing ou transitivo.

- Se $p \in T^{2}$ e $q \in W^{u}(0)$. então para qualquer $n . d\left(o^{n}(p) . \phi^{n}(q)\right) \geq I_{u}=\delta$. Portanto. o tem dependencia sensitiva em condiçòes iniciais. 
Então o difeomorfismo de Anosor no toro $T^{2}$ í caótico.

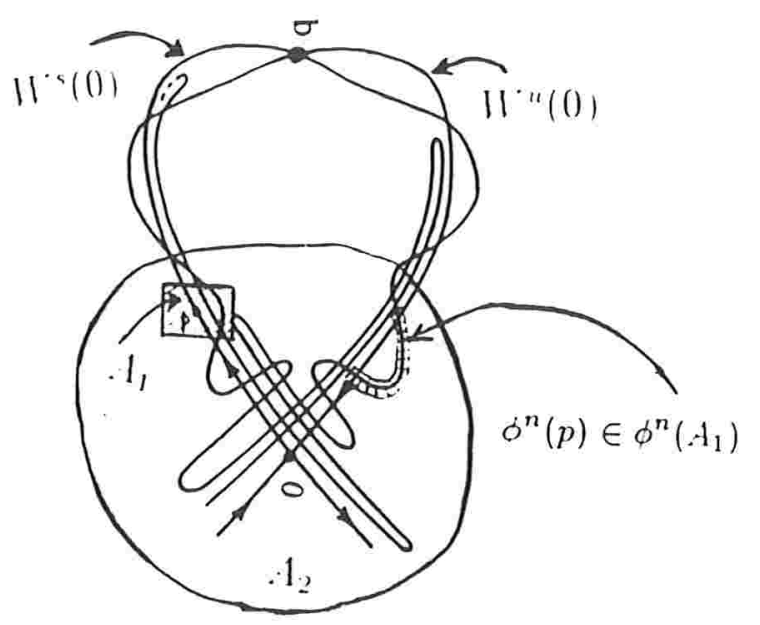

Figura 1.6: As vizinhanças $A_{1}, A_{2}$ e $\phi^{n}\left(A_{1}\right)$ 


\section{Capítulo 2}

\section{Teoria de conjuntos controláveis}

Lim sistema de controle num espaço de fases é um conjunto completo de vetores em cada ponto do espaço e não somente apenas um vetor velocidade. como nos sistemas evolutivos usuais. Então o problema de controle é escolher. a cada instante. um vetor velocidade desse conjunto de vetores e assim alcançar um subconjunto do espaço de fases. Vide Figura 2.1.

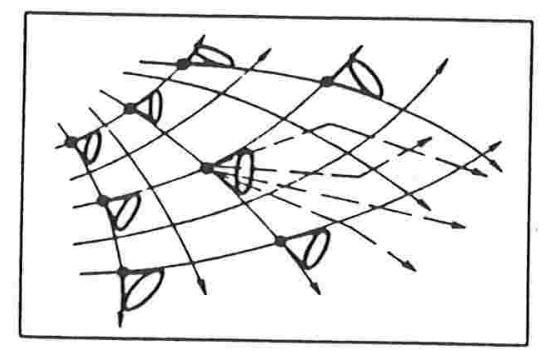

Figura 2.1

Freqüentemente. sistemas de controle não linear da forma

$$
\dot{x}=\frac{\mathrm{d} x}{\mathrm{~d} t}=X_{0}(x(t))+\sum_{i=1}^{m} u_{i}(t) X_{i}(x(t)) . \quad t>0 .
$$

e

$$
x(t+1)=f(x(t) \cdot u(t)) . \quad t=0.1 \cdot 2 \ldots .
$$

para o caso de tempo contínuo e discreto, são usados para modelar o comportamento de sistemas físicos. biológicos. sociais, onde $X_{0}, X_{3} \ldots X_{m}$ são uma família de campos de vetores de classe $C^{x}$ definidos numa variedade $M=M I^{n}$. paracompacta. conexa de 
dimensão $n \geq 1$ de classe $C^{\infty}$ e $f: M \times U \rightarrow M$ uma função que tem uma extensão diferenciável para $M \times \tilde{U}$ tal que $\tilde{U}$ é um conjunto aberto contendo $U$ definido na Seção 1.4 , onde para cada $u \in U$ a aplicação $f_{u}: M \rightarrow M$ tal que $f_{u}(x)=f(x . u)$ é um difeomorfismo global, $x$ e $u$ tomam valores em $M$. Além disso, suponhamos que quando $M$ é compacto, $\forall u \in \mathcal{U} . \forall x \in M$ tem uma única soluşão global $\varphi(t . x, u), t \in G$, tal que $\varphi_{u}(0 . x)=x$, isto é, existe um único $\varphi_{u}: \mathbb{R} \times M \rightarrow M$ que é Lipchitz $\forall t \in G$. A controlabilidade do caso contínuo podemos estudar localmente, ou seja, se $p \in M$, então existe uma vizinhança de $p, V_{p}$, tal que $\forall x, y \in V_{p}$, existe um controle $u(t)$ e um tempo finito de maneira que $\varphi(t, 0, x, u)=y$; ou globalmente, isto é, sejam $x, y \in M$, dizemos que $x$ é controlável globalmente até $y$ em tempo $t$, se existe um controle admissível $u(t)$ tal que $\varphi(t, 0, x, u)=y$. Um dos fatos centrais em controlabilidade de tempo contínuo é a seguinte propriedade, válida para sistemas analíticos, com a condição do posto da álgebra de Lie. Vide [15].

$$
\operatorname{int} O^{+}(x) \neq \phi \quad \text { em } \quad O_{t \in G}^{+}(x) \cup O_{t \in G}^{-}(x),
$$

que também pode ser estabelecido em geral para sistemas suaves, através de assunções apropriadas algébricas de Lie [2].

Assim, para tempo contínuo, o espaço estado pode ser particionado em subvariedades invariantes (que desempenham um papel cada vez mais importante no desenvolvimento da teoria de controle não linear) e dentro de cada subvariedade podem atingir um conjunto aberto de cada estado.

Em contraste (2.2) para sistemas discretos tem sido estabelecido até agora somente para casos particulares como: (a) se a órbita de $x$ é compacta; (b) se a aplicação $f$ é racional em estados e controles; (c) quando $x$ é um ponto de equilíbrio de $f$ (e o sistema é analítico e o conjunto de valores de controle é conexo).

Este capítulo será dedicado a definir os conjuntos controláveis, contínuo e discreto (simultaneamente) e ao estudo de suas propriedades usadas no presente trabalho. Estes 
conjuntos são essencialmente onde a "quasi atingibilidade" é válida, ou seja, dados $x, y$ elementos de tais conjuntos controláveis, partindo do ponto $x$ existem um controle e um tempo, podemos atingir qualquer vizinhança de $y$.

\subsection{O conceito de conjuntos controláveis}

Primeiro vamos definir as questões relativas à teoria de controle.

Definição 2.1.1 Dados $x, y \in M$. as órbitas positiva e negativa de (2.1) para o tempo $t>0, t \in G$ são

$$
\begin{aligned}
& O_{t \in G}^{+}(x)=\{y \in M, \exists u \in \mathcal{U} \text { tal que } \varphi(t, x, 0, u)=y\} \\
& O_{t \in G}^{-}(x)=\{y \in M, \exists u \in \mathcal{U} \text { tal que } \varphi(t, y, 0, u)=x\}
\end{aligned}
$$

e os conjuntos de acessibilidade, respectivamente, são

$$
O^{+}(x)=\bigcup_{t \in G} O^{+}(x) \quad \text { e } \quad O^{-}(x)=\bigcup_{t \in G} O^{-}(x)
$$

Poderíamos ainda definir $\overline{O^{+}(x)}=\left\{y \in M, \forall \delta>0, \exists u_{\delta} \in \mathcal{U}\right.$ tal que $d\left(\varphi\left(t, x, 0, u_{\delta}\right), y\right)<$ $\delta\}$, isto é, $\overline{O^{+}(x)}$ é nada mais nada menos que o fecho de $O^{+}(x)$ definido na linha anterior.

O sistema (2.1) é completamente controlável se $O^{+}(x)=M, \forall x \in M$.

Exemplo 1: O movimento de um nadador numa corrente com campo velocidade $(2,0)$ é discreto pelo sistema de controle

$$
\begin{aligned}
& \dot{x}=2+\cos u \\
& \dot{y}=\operatorname{sen} u
\end{aligned}
$$

onde $u$ é um ângulo circular. O conjunto de pontos atingíveis em tempo $t$ de um ponto $\left(x_{0}, y_{0}\right)$ é um círculo de raio $|t|$ centrado em $\left(x_{0}+2 t, y_{0}\right)$. A união destes círculos para todo $t \geq 0(t \leq 0)$ é a órbita positiva (negativa) do ponto. Vide figura 2.2 . 


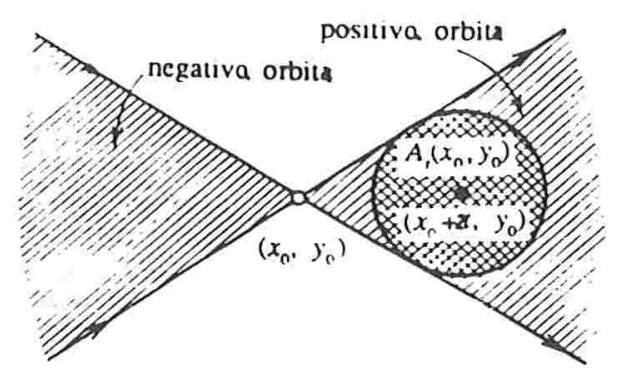

Figura 2.2

Agora vamos definir regiões nas quais o sistema (2.1) é "aproximadamente" controlável, chamados conjuntos controláveis. No caso discreto poderíamos pensar como conjuntos onde é possível chegar arbitrariamente próximo de um ponto a outro; esta definição foi introduzida por W. Kliemann.

Definição 2.1.2 (Conjunto controlável) Dizemos que $C \subset M, C \neq \emptyset$ é um conjunto controlável de (2.1) se:

Co1) $\overline{O^{+}(x)} \supset C, \forall x \in C$;

Co2) $\forall x \in C, \exists u \in \mathcal{U}$ tal que $\varphi(t, x, u) \in C, \forall t \in G$;

Co3) Se $C^{\prime} \supset C$, com $C^{\prime}$ satisfazendo 1) e 2), então $C^{\prime}=C$, isto é, $C$ é maximal em relação a "С".

Se $C$ satisfaz somente (Co1) e (Co2), chama-se conjunto pré-controlável, na verdade esta é uma definição fraca de conjunto controlável.

Um exemplo simples de um conjunto pré-controlável que não é conjunto controlável é dado por uma órbita periódica. De fato, se existem $x \in M, t \in \mathbb{N}, u \in U^{t}$ tais que $x=\varphi(t, x, u)$ então o conjunto $\{x, \varphi(1, x, u), \ldots, \varphi(t-1, x, u)\}$ só satisfaz (Co1) e (Co2) (pode não satisfazer Co3). 
Os conjuntos de um ponto $\{x\} \subset M^{1}$ são considerados conjuntos controlá veis somente se eles são pontos fixos de (2.1). isto é, $X_{i}(x)=0=f(x, \cdot), \forall i=0,1, \ldots m$. É preciso observar também que na verdade existem duas definições de um conjunto controlável. Em uma outra definição é exigida int $C \neq \emptyset$ no lugar da condição (Co2), elas são iguais quando é assumido em nossa definição a propriedade de acessibilidade local (que será definido na Seção 2.3). A definição alternativa foi usada sucessivamente por Albertini-Sontag [1] para o caso discreto, por San Martin-Tonelli [13] para semigrupos respectivamente, e era uma definição também de Kliemann.

OBS:

- Se o sistema (2.1) é completamente controlável, então $M=C$ é o único conjunto controlável, este é o caso trivial.

- No caso do sistema dinâmico clássico (sem controle) como sistema de controle, então os conjuntos controláveis são exatamente os conjuntos minimais.

Lembramos que $M$ é minimal se a órbita de todo elemento de $M$ for densa em $M$. De fato, escolhemos $x \in M$ e consideremo $\overline{O(x)}$. Dado $y \in \overline{O(x)}$, existe uma seqüência $\left(t_{j}\right)_{j \in \mathbb{N}} \subset G$ tal que $\varphi\left(t_{j}, x\right) \rightarrow y$ pela continuidade de $\varphi$ concluímos que $\varphi\left(t_{j}+1, x\right) \rightarrow \varphi(y)$. Portanto, $\varphi(y) \in \overline{O(x)}$ e $\overline{O(x)}$ resulta $\varphi$-invariante. Logo, $\overline{O(x)}=M$. A recíproca é imediata.

A seguir exibimos alguns exemplos de conjuntos controláveis.

Exemplo 2: Seja $M=S^{1}$ parametrizado pelo ângulo $\eta \in[0,2 \pi]$. Consideremos o sistema de controle

$$
\left[\begin{array}{c}
\dot{x}(t) \\
\dot{y}(t)
\end{array}\right]=\left(\begin{array}{cc}
1 & u_{1}(t) \\
u_{1}(t) & u_{2}(t)
\end{array}\right)\left(\begin{array}{c}
x(t) \\
y(t)
\end{array}\right), \quad t \in \mathbb{R} .
$$

\footnotetext{
${ }^{1}$ Como decorre da definição.
} 
Projetando estes campos de vetores no círculo $\mathbb{S}^{1}=M$ obtemos

$$
f\left(u_{1}, u_{2}, \eta\right)=\dot{\eta}=-u_{1} \operatorname{sen}^{2} \eta+\left(u_{2}(t)-1\right) \operatorname{sen} \eta \cos \eta+u_{1}(t) \cos ^{2} \eta
$$

com $u=\left(u_{1}, u_{2}\right) \in \mathcal{U}:=\left\{u: \mathbb{R} \rightarrow U \subset \mathbb{R}^{2}\right.$ mensurável $\}$, com $U=\left[0, \frac{1}{2}\right] \times[1,2]$. Observemos primeiro para controles constantes $\|_{1} \equiv \alpha, u_{2} \equiv \beta$.

Os autovalores $\lambda_{1,2}$ de $\left(\begin{array}{ll}1 & \alpha \\ \alpha & \beta\end{array}\right)$ são dados por $(1-\lambda)(\beta-\lambda)-a^{2}=\lambda^{2}+(1+\beta) \lambda+$ $\beta-\alpha^{2}=0$. Portanto,

$$
\lambda_{1,2}=\frac{1+\beta}{2} \pm \sqrt{\alpha^{2}-\beta+\frac{(1+\beta)^{2}}{4}} .
$$

Vamos olhar para valores diferentes de $\beta$ e $\alpha$.

1. Para $\beta=1$, temos $\lambda_{1,2}=1 \pm \alpha$, onde os autoespaços generalizados são dados por

$$
\begin{aligned}
& \text { se } \alpha=0: \mathbb{R}^{2} \\
& \text { se } \alpha>0: y= \pm x .
\end{aligned}
$$

2. Para $\beta=2$, temos $\lambda_{1,2}=\frac{2}{3} \pm \sqrt{\alpha^{2}+\frac{1}{4}}$. Os correspondentes autoespaços são dados por

$$
\begin{aligned}
& \alpha=0: y=0 \text { e } x=0, \operatorname{com} \lambda_{1,2}=1,2, \\
& \alpha>0: y=\left(\frac{1}{2 \alpha} \pm \sqrt{1+\frac{1}{4 \alpha^{2}}}\right) x .
\end{aligned}
$$

3. Para $\beta \in[1,2]$ e $\alpha=\frac{1}{2}$ temos sempre dois autovalores reais $\lambda_{1}>\lambda_{2}$, e os correspondentes autoespaços são da forma

$$
\begin{aligned}
& \left.V_{1}=c\left(\begin{array}{l}
x \\
y
\end{array}\right): c \in \mathbb{R}, \text { com } y=a x \text { para algum } a>1\right\}, \\
& \left.V_{2}=c\left(\begin{array}{l}
x \\
y
\end{array}\right): c \in \mathbb{R}, \text { com } y=-b x \text { para algum } b<1\right\} .
\end{aligned}
$$




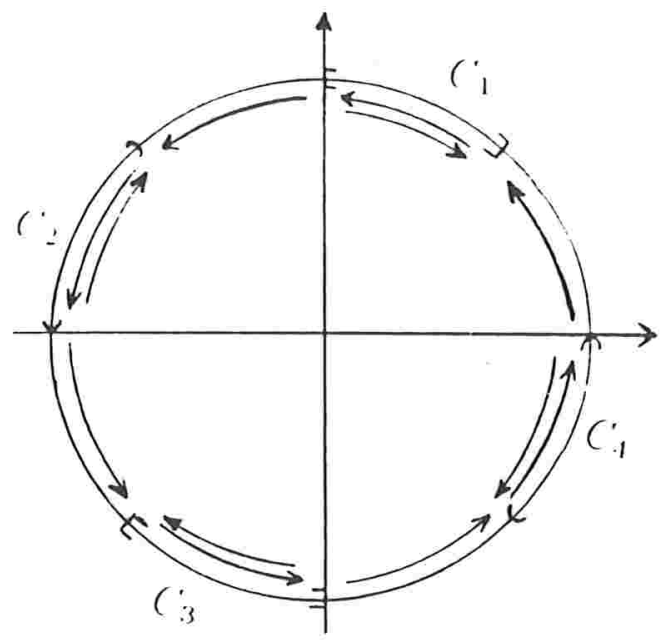

Figura 2.3: Conjuntos de controle e dinámica para o sistema (2.3)

Então obtemos as seguintes regiōes de controlabilidade:

$$
C_{1}=\left[\frac{\pi}{4}, \frac{\pi}{2}\right], \quad C_{2}=\left(\frac{3 \pi}{4}, \pi\right), \quad C_{3}=C_{1}+\pi, \quad C_{4}=C_{2}+\pi
$$

Exemplo 3: Agora vamos considerar o seguinte sistema de controle em $M=\mathbf{S}^{1}$.

$$
\dot{x}=-\operatorname{sen}^{2} x+b \cos ^{2} x-u \cos ^{2} x:=f(u, x) . \quad x \in \mathbb{R} \bmod 2 \pi, b>0 .
$$

$\operatorname{com} U=[B, b] \subset \mathbb{R}$.

Vamos calcular os pontos críticos

$$
\begin{aligned}
& -\operatorname{sen}^{2} x+b \cos ^{2} x-u \cos ^{2} x=0 \\
& \cos ^{2} x-1+(b-u) \cos ^{2} x=0 \Leftrightarrow(1-u+b) \cos ^{2} x=1 \Leftrightarrow \cos x=\frac{1}{\sqrt{1-u+b}} \\
& \text { e } \quad \operatorname{sen} x=\sqrt{1-\frac{1}{1-u+b}}=\sqrt{\frac{b-u}{1-u+b}} . \tan x=\sqrt{b-u} .
\end{aligned}
$$

Então temos os seguintes conjuntos controláveis $C_{1}=\left[0 \cdot \arctan (b-B)^{1 / 2}\right] \cdot C_{2}=(\pi-$ $\left.\arctan (b-B)^{1 / 2} \cdot \pi\right) \cdot C_{3}=C_{1}+\pi \cdot C_{4}=C_{2}+\pi$. 


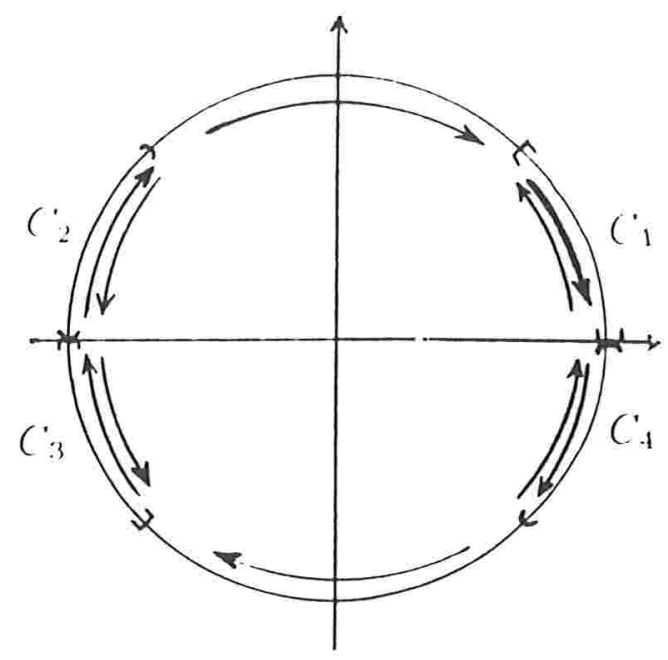

Figura 2.4: Conjuntos de controle e dinâmicas para o sistema (2.5).

¿o próximo exemplo mostraremos que para $\Lambda /$ não compacto pode não existir conljuntos controláveis.

Exemplo 4: Vamos considerar agora nosso sistema de controle em $M=\mathbb{R}$. Seja $\dot{x}=$ $X_{0}(x)+u X_{1}(x)=2+u\left(x^{2}-1\right), \operatorname{com} U=[A, B] \subset[0, \infty)$. Os pontos críticos são do tipo $x_{1.2}=\sqrt{1-2 / u}$.

- Se $B<2$, então não temos conjuntos controláveis e $\varphi(t, x, u) \rightarrow \infty$, quando $t \rightarrow \infty$. $\forall t \in \mathbb{R}, \forall u \in \mathcal{U}$.

- Se $A<2, B \geq 2$, então tem-se um único conjunto controlável

$$
C=[-\sqrt{1-2 / B}, \sqrt{1-2 / B}]
$$

- Se $A \geq 2$. então temos dois conjuntos controláveis

$$
\begin{aligned}
& C_{1}=(\sqrt{1-2 / A} \cdot \sqrt{1-2 / B}) \\
& C_{2}=[-\sqrt{1-2 / B} \cdot-\sqrt{1-2 / A}] . \quad \text { onde } C_{1}<C_{2} .
\end{aligned}
$$




\subsection{Propriedades de conjuntos controláveis}

Nesta seção estudamos as propriedades gerais de conjuntos controláveis. Uma propriedade importante da órbita positiva, que freqüentemente utilizaremos neste capítulo é o seguinte

Teorema 2.2.1 Para o sistema de controle (2.1). sejam $x_{1}, x_{2}$ pontos quaisquer da variedade $M$ e se $x_{2} \in \overline{O^{+}\left(x_{1}\right)}$, entâo $\overline{O^{+}\left(x_{2}\right)} \subset \overline{O^{+}\left(x_{1}\right)}$.

Demonstração: Primeiro lembremos o fecho da órbita positiva $\overline{O^{+}(x)}=\{y \in M, \forall \epsilon>$ $0, \exists u \in \mathcal{U}$ tal que $d(\varphi(t, x, u), y)<\epsilon\}$.

Seja $y \in \overline{O^{+}\left(x_{2}\right)}$, vamos fixar $\epsilon>0$ e uma métrica $d$ em $M$, então existem $t \in G$ e $u \in \mathcal{U}$ tais que $d\left(\varphi\left(t, x_{2}, u\right), y\right)<\epsilon / 2$. Como $\varphi(t, \cdot, u)$ é contínua, existe uma vizinhança de $x_{2}, V_{x_{2}}$, de maneira que $d(\varphi(t, z, u), y)<\epsilon, \forall z \in V_{x_{2}}$.

Agora, por hipótese, existem $s \in G, v \in \mathcal{U}$ tais que $z=\varphi\left(s, x_{1}, v\right) \in V_{x_{2}}$, então $d\left(\varphi\left(s, x_{1}, v\right), x_{2}\right)<\epsilon / 2$. Logo, $\varphi\left(t, \varphi\left(s, x_{1}, v\right), u\right) \in V_{x_{2}} \Rightarrow \varphi\left(t+s, x_{1}, u \wedge v\right) \in V_{x_{2}}{ }^{2}$. Então $d\left(\varphi\left(t+s, x_{1}, u \wedge v\right), y\right)<\epsilon \Rightarrow y \in \overline{O^{+}\left(x_{1}\right)}$, pois $y \in \overline{O^{+}\left(x_{2}\right)}$ e $\epsilon>0$ eram arbitrários.

Para dois pontos quaisquer no conjunto controlável $C$, qualquer trajetória ligando-os está sempre em $C$, diz a seguinte

Proposição 2.2.2 Seja $x_{1}, x_{2} \in C \subset M$ para algum conjunto controlável e para algum. $u \in \mathcal{U}$ vale que $x_{2}=\varphi\left(t, x_{1}, u\right)$, então $\varphi\left(s, x_{1}, u\right) \in C$ para $s \in[0, t]$.

Demonstração: Consideremos o conjunto $B=\left\{\varphi\left(s, x_{1}, u\right), 0 \leq s \leq t\right\}$. Vamos mostrar que $C_{1}=C \cup B$ é um conjunto pré-controlável, isto é,

(a) $C_{1} \subset \overline{O^{+}(x)}, \forall x \in C_{1}$

(b) dado $z \in C_{1}, \exists u \in \mathcal{U}$ tal que $\varphi(t, z, u) \in C_{1}$.

\footnotetext{
${ }^{2} u \wedge v$ é a concatenação de $u$ e $v$.
} 
De fato,

(a) basta mostrar as seguintes relações

(a1) $C \subset \overline{O^{+}(x)}, \forall x \in C_{1}$;

(a2) $B \subset \overline{O^{+}(x)}, \forall x \in C_{1}$.

Vamos mostrar (a1) em duas partes:

- Como $C$ é conjunto controlável, então $C \subset \overline{O^{+}(x)}, \forall x \in C$.

- Agora mostraremos que $C \subset \overline{O^{+}(x)}, \forall x \in B$.

Seja $x \in B \Rightarrow$ existem $s_{1} \in[0, t]$ t.al que $\varphi\left(s_{1}, x_{1}, u\right)=x \Rightarrow \varphi\left(t-s_{1}, x, \theta_{s_{1}} u\right)=x_{2}$, (onde $\left.\theta_{s_{1}} u=u\left(\cdot+s_{1}\right)\right) \Rightarrow x_{2} \in O^{+}(x) \Rightarrow \overline{O^{+}\left(x_{2}\right)} \subset \overline{O^{+}(x)}$, pela Proposição 2.2.1. Como $x_{2} \in C \Rightarrow C \subset \overline{O^{+}\left(x_{2}\right)} \subset \overline{O^{+}(x)}, \forall x \in B, \operatorname{logo} C \subset \overline{O^{+}(x)}, \forall x \in C_{1}$.

De maneira análoga, (a2) dividamos em duas partes:

- Vamos mostrar que $B \subset \overline{O^{+}(x)}, \forall x \in C$. Para isso, tomemos $x \in C$ e $y \in B$, logo existem $s_{2} \in(0, t]$ e $u \in \mathcal{U}$ tal que $\varphi\left(s_{2}, x_{1}, u\right)=y$.

Agora, seja uma vizinhança de $y, V_{y}$. Como $\varphi\left(s_{2}, \cdot, u\right)$ é contínua, existe uma vizinhança de $x_{1}, V_{x_{1}}$, tal que $\varphi\left(s_{2}, V_{x_{1}}, u\right) \subset V_{y}$. Além disso, $x_{1} \in C \subset \overline{O^{+}(x)}$.

$\Rightarrow$ existem $s_{3} \in(0, t]$ e $v \in \mathcal{U}$ de maneira que $\varphi\left(s_{3}, x, v\right) \in V_{x_{1}}$.

$\Rightarrow \varphi\left(s_{2}, \varphi\left(s_{3}, x, v\right), u\right) \in V_{y}$.

$\Rightarrow \varphi\left(s_{2}+s_{3}, x, u \wedge v\right) \in V_{y} \Rightarrow y \in O^{+}(x) \Rightarrow y \in \overline{O^{+}(x)}, \forall x \in C$.

Portanto, $B \subset \overline{O^{+}(x)}, \forall x \in C$.

- Resta mostrar que $B \subset \overline{O^{+}(x)}, \forall x \in B$.

Sejam $x \in B$ e $y \in B ; y \in \overline{O^{+}(x)}$. Como $x \in B$, então existe $s_{4} \in[0, t]$ tal que $\varphi\left(s_{4}, x_{1}, u\right)=x, \operatorname{logo} x_{2} \in O^{+}(x) \Rightarrow \overline{O^{+}\left(x_{2}\right)} \subset \overline{O^{+}(x)}$. Como $x_{2} \in C \Rightarrow$ $C \subset \overline{O^{+}\left(x_{2}\right)} \subset \overline{O^{+}(x)} \Rightarrow C \subset \overline{O^{+}(x)}, \forall x \in B$. 
(b) é imediato. Portanto fica provado o resultado.

Proposição 2.2.3 Seja $C \subset M$ um conjunto controlável, int $C \neq \emptyset$. e $x_{1}, x_{2} \in \operatorname{int} C$ e para algum controle $u \in \mathcal{U}$ tem-se $x_{2}=\varphi\left(t, x_{1}, u\right)$. Então $\varphi\left(s, x_{1}, u\right) \in \operatorname{int} C$. para $s \in[0, t]$.

Demonstração: Pela Proposição $2.2 .2 \varphi\left(s, x_{1}, u\right) \in C$. Suponhamos que existem $s \in$ $[0, t]$ e $u \in \mathcal{U}$ tais que $\varphi\left(s, x_{1}, u\right) \in \partial C$, fazemos $\varphi\left(s, x_{1}, u\right)=y$. Sabemos que existem $t_{1} \in[0, t]$ e $u_{1} \in \mathcal{U}$ tal que $\varphi\left(t_{1}, y, u_{1}\right)=x_{2}$, mas $x_{2}=\varphi\left(t, x_{1}, u\right)=\varphi\left(t-s+s, x_{1}, u\right)=$ $\varphi\left(t-s, x_{1}, \theta_{s} \theta_{-s} u\right)=\varphi\left(t-s, \varphi\left(s, x_{1}, u\right), \theta_{-s} u\right)=\varphi\left(t_{1}, y, u_{1}\right)$. Como $x_{2} \in \operatorname{int} C$. então existe uma vizinhança de $y, \mathcal{N}_{y}$, de maneira que $\varphi\left(t_{1}, \mathcal{N}_{y}, u_{1}\right) \subset \operatorname{int} C$.

Agora, como $\varphi(s, \cdot, u)$ é um difeomorfismo local, pelo fato de ser um fluxo, então existe uma vizinhança de $x_{1}, \mathcal{N}_{x_{1}}$ tal que $\varphi\left(s, \mathcal{N}_{x_{1}}, u\right)$ contém $y$, isto é, $V_{y}=\varphi\left(s, \mathcal{N}_{x_{1}}, u\right)$.

Portanto, $V_{y} \cap \mathcal{N}_{y}$ é um aberto que contém $y$, ou seja, é uma vizinhança de $y$. Como estamos supondo $y \in \partial C \Rightarrow \exists z \in V_{y} \cap N_{y}$ tal que $z \notin C$. Mas por um lado $z=\varphi\left(s, z_{1}, u\right)$ com $z_{1} \in \mathcal{N}_{x_{1}} \subset$ int $C$. Por outro lado, $\varphi\left(t_{1}, z, u_{1}\right)=z_{2} \in \operatorname{int} C$, daí $\varphi\left(t_{1}, \varphi\left(s, z_{1}, u\right), u_{1}\right)=$ $z_{2}, \Rightarrow \varphi\left(t_{1}+s, z_{1}, u_{1} \wedge u\right)=z_{2} \Rightarrow \varphi\left(t, z_{1}, u_{1} \wedge u\right)=z_{2} \Rightarrow \varphi\left(s, z_{1}, u_{1} \wedge u\right) \in C . \operatorname{Em}$ particular, $z \in C$, contradição! Portanto, $z \notin \partial C \Rightarrow y \in$ int $C$, o que prova a proposição.

Teorema 2.2.4 Seja $C \subset M$ um conjunto controlável, int $C \neq \emptyset$. Então $\bar{C}=\overline{\operatorname{int} C}$.

Demonstração: Seja $x \in C$ e uma vizinhança de $x, W_{x}$, vamos mostrar que $W_{x} \cap$ $\operatorname{int} C \neq \emptyset$. Tomemos um ponto arbitrário $y \in \operatorname{int} C$. Como $C$ é conjunto controlável, $\Rightarrow C \subset \overline{O^{+}(x)}, \forall x \in C . \Rightarrow y \in O^{+}(x)$, logo existem $t \in G$, um controle $u \in \mathcal{U}$ tal que $\varphi(t, x, u)=z \operatorname{com} z \in \operatorname{int} C$. Agora tomamos uma vizinhança de $z, V_{z}, V_{z} \subset C \Rightarrow$ como $\varphi(t, \cdot, u)$ é contínua, existe uma vizinhança de $x, V_{x}, \operatorname{com} \varphi(t, y, u) \in V_{z}, \forall y \in V_{x}$, então $\varphi(t, y, u) \in \operatorname{int} C$. Consideremos $V=V_{x} \cap W_{x}$ e tomemos $y^{\prime} \in \operatorname{int} C$. Como $x \in \overline{O^{+}\left(y^{\prime}\right)} \Rightarrow \exists t^{\prime} \in G, u^{\prime} \in \mathcal{U}$ tais que $\bar{x}=\varphi\left(t^{\prime}, y^{\prime}, u^{\prime}\right) \in V, \operatorname{logo} \bar{x} \in V_{x}, \varphi(t, \bar{x}, u) \in \operatorname{int} C$. Agora $\varphi\left(t, \varphi\left(t^{\prime}, y^{\prime}, u^{\prime}\right), u\right) \in \operatorname{int} C \Rightarrow \varphi\left(t+t^{\prime}, y^{\prime}, u \wedge u^{\prime}\right) \in \operatorname{int} C$. 
Sejam $y^{\prime}$ e $\varphi\left(t+t^{\prime}, y^{\prime} \cdot u \wedge u^{\prime}\right)$ como sendo $x_{1} \cdot x_{2}$ da Proposição 2.2.2. Então $\bar{x}=$ $\varphi\left(t^{\prime}, y^{\prime}, u^{\prime}\right) \in \operatorname{int} C \Rightarrow V \cap \operatorname{int} C \neq \emptyset \Rightarrow \Psi_{x} \cap \operatorname{int} C \neq \emptyset$. Portanto. fica provado o teorema.

O teorema seguinte diz que os fechos das órbitas positivas de dois pontos contidos no mesmo conjunto controlável coincidem.

Teorema 2.2.5 Para um conjunto controlável $C \subset M$ tem-se que $\overline{O^{+}\left(x_{1}\right)}=\overline{O^{+}\left(x_{2}\right)}$, $\forall x_{1}, x_{2} \in C$

Demonstração: Por hipótese, $C$ é conjunto controlável. Então,

$C \subset O^{+}\left(x_{1}\right)$ se $x_{2} \in \overline{O^{+}\left(x_{1}\right)}$, pelo Teorema $2.2 .1, \overline{O^{+}\left(x_{2}\right)} \subset \overline{O^{+}\left(x_{1}\right)}$

$C \subset O^{+}\left(x_{2}\right)$ se $x_{1} \in \overline{O^{+}\left(x_{2}\right)}$, pelo Teorema $2.2 .1, \overline{O^{+}\left(x_{1}\right)} \subset \overline{O^{+}\left(x_{2}\right)}$.

Portanto, $\overline{O^{+}\left(x_{1}\right)}=\overline{C^{+}\left(x_{2}\right)}, \forall x_{1}, x_{2} \in C$.

Uma conseqüência do teorema anterior é o seguinte

Corolário 2.2.6 Para cada $x \in C \subset M$, onde $C$ é conjunto controlável e cada $\tau \in \mathbb{N}$, temos que

$$
\overline{O^{+}(x)}=\overline{\bigcup_{t=\tau}^{\infty} O_{t}^{+}(x)}
$$

Demonstração: (i) é claro que $\overline{O^{+}(x)} \supset \overline{\bigcup_{t=\tau}^{\infty} O_{t}^{+}(x)}$.

(ii) Como $C$ é conjunto controlável, então para cada $\tau \in G$ existe $u \in \mathcal{U}$ tal que $\varphi(\tau, x, u) \in C$. Tomemos $x$ e $\varphi(\tau, x, u)$ como sendo $x_{1}$ e $x_{2}$ do Teorema 2.2.5, então

$$
\overline{O^{+}(x)}=\overline{O^{+}(\varphi(\tau, x, u)} \subset \overline{\bigcup_{t=\tau}^{\infty} O_{t}^{+}(x)} \text {. }
$$

Proposição 2.2.7 Seja $M$ uma variedade e consideremos $C_{1}, C_{2} \subset M$ conjuntos controláveis. Então $C_{1} \cap C_{2}=\emptyset$ ou $C_{1}=C_{2}$. 
Demonstração: Vamos mostrar que $C_{1} \cup C_{2}$ é um conjunto controlável. Para isto. primeiro:

- Suponhamos que $C_{1} \cap C_{2} \neq \emptyset$, isto é, $\exists x \in C_{1} \cap C_{2}$. Seja $y \in C_{1}$, como $x \in \overline{O^{+}(y)}$ e $C_{2} \subset \overline{O^{+}(x)}$, então aplicando o Teorema 2.2.1, temos $\overline{O^{+}(x)} \subset \overline{O^{+}(y)} \Rightarrow C_{2} \subset$ $\overline{O^{+}(y)}, \forall y \in C_{1}$.

Analogamente, seja $y \in C_{2}$, como $x \in \overline{O^{+}(y)}$ e $C_{1} \subset \overline{O^{+}(x)}$. Novamente aplicando o Teorema 2.2.1, tem-se que $\overline{O^{+}(x)} \subset \overline{O^{+}(y)} \Rightarrow C_{1} \subset \overline{O^{+}(y)}, \forall y \in C_{2}$. Portanto. $C_{1} \cup C_{2} \subset \overline{O^{+}(y)}, \forall y \in C_{1} \cup C_{2}$.

- A segunda propriedade de conjunto controlável é imediata pela definição.

- Como $C_{1}$ e $C_{2}$ satisfazem a propriedade de maximalidade, então $C_{1}=C_{1} \cup C_{2}=C_{2}$.

Proposição 2.2.8 Para cada $C^{\prime} \subset M$ subconjunto pre-controlável existe um único conjunto controlável $C \subset M$ tal que $C^{\prime} \subset \mathrm{C}$.

Demonstração: Para $C^{\prime} \subset M$ conjunto pré-controlável, vamos definir $C=\bigcup_{\alpha \in \mathbb{N}} C_{\alpha}$, onde cada $C_{\alpha}$ é conjunto pre-controlável tal que $C^{\prime} \subset C_{\alpha}$. É óbvio que $C \neq \emptyset$. Agora mostremos que $C$ é conjunto controlável.

Co1) Vamos mostrar que $C \subset \overline{O^{+}(x)}, \forall x \in C$, fixemos $\epsilon>0$ e uma métrica $d$ em $M$. Seja $y \in C$, então $y \in C_{\alpha}$ para algum $\alpha \in \mathbb{N}$. Tomemos uma vizinhança de $y, V_{y}$, e $y \in C^{\prime} \subset C_{\beta}$, logo existem $t_{1} \in G$ e $u_{1} \in \mathcal{U} \operatorname{com} \varphi\left(t_{1}, z, u_{1}\right) \in V_{y}$. Como $\varphi\left(t_{1}, \cdot, u_{1}\right)$ é contínua, existe uma vizinhança de $z, V_{z}$, tal que $\varphi\left(t_{1}, V_{z}, u_{1}\right) \subset V_{y}$. Agora, $z \in C^{\prime} \subset C_{\alpha} \ni x \Rightarrow$ existem $t_{2} \in G$ e $u_{2} \in \mathcal{U}$ de maneira que $\varphi\left(t_{2}, x, u_{2}\right) \in V_{z}$. Daí, $\varphi\left(t_{1}, \varphi\left(t_{2}, x, u_{2}\right), u_{1}\right) \in V_{y} ; \varphi\left(t_{1}+t_{2}, x, u_{1} \wedge u_{2}\right) \in V_{y} \Rightarrow d\left(\varphi\left(t_{1}+t_{2}, x, u_{1} \wedge u_{2}\right), y\right)<\epsilon$. Aplicando-se a definição de órbita fechada, tem-se que $y \in \overline{O^{\perp}(x)}, \forall x \in C$. Como $x$ e $y$ eram arbitrários, temos que $C \subset \overline{O^{+}(x)}, \forall x \in C$. 
Co2) É imediata, pois cada $x \in C$ está contido em um conjunto pré-controlável contido em $C$.

Co3) A maximalidade segue da definição de $C$. Portanto, existe um conjunto controlável.

Finalmente, a unicidade temos de: dois conjuntos controláveis são disjuntos ou iguais.

Lema 2.2.9 Sejam $C_{1}^{\prime}, C_{2}^{\prime} \subset M$ conjuntos pre-controláveis, $C_{1}^{\prime} \cap C_{2}^{\prime} \neq \emptyset$, então $C_{1}^{\prime} \cup C_{2}^{\prime}$ é um conjunto pré-controlável.

Demonstração: Devemos mostrar que a união satisfaz as duas propriedades de controlabilidade.

- Pela Proposição 2.2.8, sejam $C_{1}, C_{2}$ conjuntos controláveis unicamente determinados contendo $C_{1}^{\prime}$ e $C_{2}^{\prime}$, respectivamente. Por hipótese, segue que $C_{1} \cap C_{2} \neq \emptyset$, então $C_{1}=C_{2}$. Assim, $\forall \xi \in C_{1}^{\prime} \cup C_{2}^{\prime}$, segue que $C_{1}^{\prime} \cup C_{2}^{\prime} \subset C_{1} \subset \overline{O^{+}(\xi)}$.

- A condição (Co2) da definição é obviamente satisfeita para $C_{1}^{\prime} \cup C_{2}^{\prime}$ desde que já foi satisfeita para $C_{1}^{\prime}$ e $C_{2}^{\prime}$.

Para encerrar esta seção, vamos definir uma ordem na família de conjuntos controláveis.

Definição 2.2.1 Sejam $C_{1}, C_{2} \subset M$ conjuntos controláveis. Dizemos que $C_{1}$ é majorado por $C_{2}$ e denotamos por $C_{1} \leq C_{2}$, se existem $x \in C_{1}$ e $y \in C_{2}$ tais que $y \in \overline{O^{+}(x)}$, isto é, existe $x \in C_{1} \operatorname{com} \overline{O^{+}(x)} \cap C_{2} \neq \emptyset$.

A relação " $\leq$ " é uma ordem parcial no conjunto $\{C \subset M ; C$ é um conjunto controlável de (2.1)\}, isto é, reflexiva, anti-simétrica e transitiva que é o que será provado na seguinte Proposição 2.2.ĩ Seja $C \subset M$ um conjunto controlável, então " $\leq$ " é uma ordem parcial nos conjuntos controláveis do sistema (2.1). 
Demonstração: (i) $C \leq C$ segue imediatamente da Definição 2.2.1.

(ii) Se $C_{1} \leq C_{2} \Rightarrow \exists x_{1} \in C_{1}, x_{2} \in C_{2}$, tais que $x_{2} \in \overline{O^{+}\left(x_{1}\right)}$ e $C_{1} \leq C_{2} \Rightarrow \exists y_{2} \in C_{2}$, $y_{1} \in C_{1}$ tais que $y_{1} \in \overline{O^{+}\left(y_{2}\right)}$. Como $x_{j}, y_{j} \in C_{j}$, para $j=1.2$. temos que $x_{j} \in$ $O^{+}\left(y_{j}\right)$ e $y_{j} \in \overline{O^{+}\left(x_{j}\right)} \Rightarrow y_{2} \in \overline{O^{+}\left(x_{1}\right)}$ e $x_{1} \in \overline{O^{+}\left(y_{2}\right)}$. Portanto, $x_{1}, y_{2}$ estão no mesmo conjunto controlável pela definição de conjunto controlável. Logo, $C_{1}=C_{2}$.

(iii) Se $C_{1} \leq C_{2} \Rightarrow \exists x \in C_{1}, y_{1} \in C_{2}$ tal que $y_{1} \in \overline{O^{+}(x)}$ e $C_{2} \leq C_{3} \Rightarrow \exists y_{2} \in C_{2}, z \in C_{3}$ tal que $z \in \overline{O^{+}\left(y_{2}\right)}$. Como $y_{1}, y_{2} \in C_{2}$, sabemos que $y_{2} \in \overline{O^{+}\left(y_{1}\right)}$. Logo, usando dependência contínua em valores iniciais, temos que $z \in \overline{O^{+}(x)}$, portanto $C_{1} \leq C_{3}$.

\subsection{Conjuntos controláveis invariantes}

Nesta seção vamos estudar os conjuntos controláveis invariantes. Primeiro apresentamos sua definição, depois provamos suas propriedades mais importantes, tanto em uma variedade paracompacta, como em uma variedade compacta.

Definição 2.3.1 Dizemos que um conjunto controlável $C \subset M$ é invariante para o sistema (2.1) se:

$$
\bar{C}=\overline{O^{+}(x)}, \quad \forall x \in C
$$

Definição 2.3.2 O sistema (2.1) é acessivel localmente quando

$$
\operatorname{int} O^{+}(x) \neq \emptyset \quad e \quad \operatorname{int} O^{-}(x) \neq \emptyset, \quad \forall x \in M, \quad \forall t \in G
$$

Nos resultados seguintes vamos supor que o sistema é localmente acessível. Na proposição que segue vamos dar sentido às palavras "os conjuntos controláveis invariantes contêm seus pontos aderentes". 
Proposição 2.3.1 Seja $C \subset M$ um conjunto controlável invariante para (2.1). $\operatorname{int} O^{+}(x) \neq$ Ø. Entâo C é fechado em $M$.

Demonstração: Vamos provar que $\bar{C}=C$, isto é, $C \subset \bar{C}$ e $\bar{C} \subset C$. Para o primeiro caso nada temos a fazer. Para provar a segunda parte, levamos em conta que $C$ é invariante, logo: seja $y \in \bar{C}$, então $\overline{O^{+}(y)} \subset \bar{C}$.

Suponhamos por absurdo que existem $t_{0} \in G, u_{0} \in \mathcal{U}$ tal que $y_{0}=\varphi\left(t_{0}, y, u_{0}\right) \notin \bar{C}$. Portanto, existe uma vizinhança de $y_{0}, V_{y_{0}}$, tal que $V_{y_{0}} \cap \bar{C} \neq \emptyset$. Como $\varphi\left(t_{0}, \cdot, u_{0}\right)$ é contínua, então existe uma vizinhança de $y, V_{y}, \operatorname{com} \varphi\left(t_{0}, V_{y}, u_{0}\right) \subset V_{y_{0}}$. Como $V_{y} \cap C \neq \emptyset$, então existe $y_{1} \in V_{y} \cap C$ tal que $\varphi\left(t_{0}, y_{1}, u\right) \in V_{y_{0}}$. Portanto, $\varphi\left(t_{0}, y_{1}, u_{0}\right) \notin \bar{C}$, que contradiz a invariança de $C$ e vale que $\overline{O^{+}(y)} \subset \bar{C}$.

Além disso, $\operatorname{int} O^{+}(y) \neq \emptyset, \forall y \in \bar{C}$. Portanto, int $O^{+}(y) \cap C \neq \emptyset$. Logo, existe $\bar{x} \in \operatorname{int} O^{+}(y) \cap C$. Por sutro lado, $\bar{C}=\overline{O^{+}(\bar{x})}$, então $y \in C$.

De particular importância são os conjuntos controláveis com interior, pois em seu interior a controlabilidade exata em tempo finito é válida.

Corolário 2.3.2 Consideremos $C \subset M$ um conjunto controlável invariante para (2.1), $\operatorname{int} O^{+}(x) \neq \emptyset$. Então, $\operatorname{int} C \neq \emptyset$.

Demonstração: Como $C$ é conjunto controlável invariante,

$$
\Rightarrow \overline{O^{+}(x)} \subset \bar{C}=C, \quad \forall x \in C, \text { pois } C \text { é fechado. }
$$

Logo, int $\overline{O^{+}(x)} \subset \operatorname{int} C, \forall x \in C$ e como por hipótese int $O^{+}(x) \neq \emptyset$, então $\emptyset \neq \operatorname{int} \overline{O^{+}(x)} \subset$ $\operatorname{int} C, \forall x \in C$. Portanto, int $C \neq \emptyset$.

O resultado seguinte mostra que sempre acontece que os conjuntos controláveis invariantes contêm a órbita positiva de $x$, para todo $x$ que está em $C$. 
Lema 2.3.3 Seja C $\subset$ M um conjunto controlável invariante para (2.1). Entào $O^{+}(x) \subset$ C. $\forall x \in C$.

Demonstração: Como $C$ é invariante, temos $\bar{C} \supset \overline{O^{+}(x)}, \forall x \in C$. Portanto, $\bar{C} \supset O^{+}(x)$, $\forall x \in C$.

Sabemos que $C$ é fechado, pela Proposição 2.3.1 então $C \supset O^{+}(x), \forall x \in C$.

O resultado que segue é uma propriedade de interesse para sistemas discretos (mas vale para sistemas não discretos).

Lema 2.3.4 Consideremos o sistema de controle (2.1), $\operatorname{com} \operatorname{int} O^{+}(x) \neq \emptyset$ e se $\forall x \in$ $M, O_{1}^{+}(x)$ é conexo, então um conjunto controlável $C \subset M$ fechado com int $C \neq \emptyset e ́$ invariante.

Demonstração: (i) Como $C$ é conjunto controlável, então $C \subset \overline{O^{+}(x)}, \forall x \in C$, portanto $\bar{C} \subset \overline{O^{+}(x)}, \forall x \in C$.

(ii) Vamos mostrar que $\overline{O^{+}(x)} \subset \bar{C}, \forall x \in C$ ou $O^{+}(x) \subset C, \forall x \in C$. Como $C$ é fechado, então $C=\bar{C}$. Agora tomemos um ponto arbitrário $y \in C$, logo existem um tempo $t \in \mathbb{N}$ e um controle $u \in U^{\mathbb{Z}}$ tais que $\varphi(t, y, u) \in \operatorname{int} C$. Como $\varphi(t, \cdot, u)$ é contínua, então existe uma vizinhança de $y, V_{y}, \operatorname{com} \varphi\left(t, V_{y}, u\right) \subset \operatorname{int} C$, logo existe uma aberto $V \supset C$ tal que $O^{+}(x) \cap \operatorname{int} C \neq \emptyset, \forall x \in V$ e daí $C \subset \overline{O^{+}(x)}, \forall x \in V$, pois $y \in O^{+}(x) \Rightarrow O^{+}(y) \subset O^{+}(x) \Rightarrow \overline{O^{+}(y)} \subset \overline{O^{+}(x)} \Rightarrow C \subset \overline{O^{+}(y)} \subset \overline{O^{+}(x)}$.

Agora suponhamos que existem um ponto $x \in C$ e um controle $u \in U^{\mathbb{Z}}$ com $\varphi(1, x, u) \notin C$. Como $C \subset \overline{O^{+}(x)}$, então existe $y \in O^{+}(x) \cap C$, logo pela Proposição 2.2.2 existe um controle $v \in U^{\mathbb{Z}}$ tal que $\varphi(1, x, v) \in C$, daí $\overline{O_{1}^{+}(x)} \cap C \neq \emptyset$. Sabemos que $O_{1}^{+}(x)$ é conexo, logo $O_{1}^{+}(x) \cap V \neq \emptyset \Rightarrow z \in O_{1}^{+}(x)$ com $z \in V \backslash C$, logo existem $u \in U^{\mathbb{Z}}, t \in \mathbb{N}$ tal que $\varphi(t, z, u) \in \operatorname{int} C \Rightarrow \varphi\left(t, \varphi\left(1, x, u_{1}\right), u\right) \in \operatorname{int} C$ 
$\Rightarrow \varphi\left(t+1, x, u_{1} \wedge u\right) \in \operatorname{int} C \Rightarrow z \in C$, logo pela maximalidade $\varphi(t, x, u) \in C$,

$\forall x \in C$, o que é absurdo! Portanto $O^{+}(x) \subset C$.

O resultado abaixo só o enunciamos aqui porque ele será importante para provar a proposição seguinte.

Teorema 2.3.5 int $O^{+}(x)$ é um subconjunto denso de $O^{+}(x)$.

Demonstração: Vide [15].

Como estamos assumindo a propriedade de acessibilidade local, a seguir provaremos a controlabilidade exata no interior do conjunto controlável $C$ (válido só no caso de sistemas em tempo contínuo).

Proposição 2.3.6 Consideremos $C \subset M$ conjunto controlável invariante para o sistema de controle (2.1). Então $O^{+}(x)=\operatorname{int} C, \forall x \in \operatorname{int} C$.

\section{Demonstração:}

(i) Vamos provar que int $C \subset O^{+}(x)$. Como $C$ é invariante, então $\overline{O^{+}(x)}=\bar{C}, \forall x \in C$, usando invariancia e as condições 2 e 3 da definição de conjunto controlável, temos que $\overline{O^{+}(x)}=C, \forall x \in C$. Logo, int $\overline{O^{+}(x)}=\operatorname{int} C$. Para ter a inclusão precisamos ainda provar que $\operatorname{int} O^{+}(x)=\operatorname{int} \overline{O^{+}(x)}$.

De fato, vamos provar que int $\overline{O^{+}(x)} \subset \operatorname{int} O^{+}(x)$. Sejam $z \in \operatorname{int} \overline{O^{+}(x)}$ e $\tilde{O}^{+}(z)$ o conjunto de todos os $z^{\prime}$ tais que $z$ é acessível a partir de $z^{\prime}$ em tempo $t \in \mathbb{R}^{+}$, então $\tilde{O}^{+}(z)$ é o conjunto acessível para o sistema de controle reverso

$$
\frac{\mathrm{d} x}{\mathrm{~d} t}=-X_{0}(x(t))-\sum_{i=1}^{m} u_{i}(t) X_{i}(x(t)) .
$$


Logo, pelo Teorema 2.3.5. int $\widetilde{O}^{+}(z)$ é denso em $\tilde{O}^{+}(z)$. Agora, como $z \in \overline{\widetilde{O}^{+}(z)}$ é válido, então $\overline{O^{+}(z)} \cap \operatorname{int} \overline{O^{+}(x)} \neq \emptyset$. Assim, temos que

$$
\operatorname{int} \tilde{O}^{+}(z) \cap \operatorname{int} \overline{O^{+}(x)} \cap O^{+}(x) \neq \emptyset
$$

Escolhemos un ponto $y$ do conjunto anterior, então $y$ é acessível de $x$ e $z$ é acessível de $y$, portanto $z$ é acessível de $x$. Isto prova

$$
\operatorname{int} \overline{O^{+}(x)} \subset O^{+}(x)
$$

Logo, int $\overline{O^{+}(x)} \subset \operatorname{int} O^{+}(x) ;$ portanto int $O^{+}(x)=\operatorname{int} \overline{O^{+}(x)}=\operatorname{int} C$. Daí, concluímos que int $C \subset O^{+}(x), \forall x \in$ int $C$.

(ii) Resta apenas provar que $O^{+}(x) \subset \operatorname{int} C, \forall x \in \operatorname{int} C$. De fato, suponhamos por absurdo que existem $t_{0} \in G$ e $u_{0} \in \mathcal{U}$ tais que $y=\varphi\left(t_{0}, x, u_{0}\right) \in \partial C$, então existe uma vizinhança de $x, V_{x} \subset \operatorname{int} C \subset C$ de maneira que $\varphi\left(t_{0}, V_{x}, u_{0}\right)$ é uma vizinhança de $y$, pois $\varphi\left(t_{0}, \cdot, u_{0}\right)$ é contínua que tem pontos de $C=\bar{C}$ e fora de $\bar{C}$, isto não pode, pois $\varphi\left(t_{0}, V_{x}, u_{0}\right) \subset \bar{C}=C$, daí, existe $\bar{x} \in V_{x}$ tal que $\varphi\left(t_{0}, \bar{x}, u_{0}\right)$ está fora de $\bar{C}$, mas $\bar{x} \in C$, logo $O^{+}(\bar{x}) \subset \bar{C}$, absurdo!

Portanto, $O^{+}(x) \subset$ int $C, \forall x \in \operatorname{int} C$.

A proposição que segue nos permitirá saber a quantidade de conjuntos controláveis.

Proposição 2.3.7 Se a variedade $M$ admite uma base enumerável de abertos e int $O^{+}(x) \neq$ $\emptyset$, então existe uma quantidade no máximo enumerável de conjuntos controláveis $C$, com $\operatorname{int} C \neq \emptyset$.

Demonstração: Seja $B_{i}$ uma base enumerável de abertos, então se $C$ é conjunto controlável com int $C \neq \emptyset$, logo existe $B_{i}$ com $B_{i} \subset$ int $C$; portanto existe uma quantidade no máximo enumerável de conjuntos controláveis $C$, com int $C \neq \emptyset$. 
Em particular, se int $O^{+}(x) \neq \emptyset \Rightarrow$ int $C \neq \emptyset$. Portanto. existe uma quantidade enumerável de conjuntos controláveis invariantes.

Com a ajuda do Lema de Zorn's vamos provar a existência de pelo menos um conjunto controlável invariante numa variedade compacta no seguinte

Lema 2.3.8 Seja $M$ uma variedade compacta, então existe pelo menos um conjunto controlável invariante em $M$.

Demonstração: Mostraremos que para cada $x_{0} \in M$ existe um conjunto controlável invariante $C_{x_{0}} \subset \overline{O^{+}\left(x_{0}\right)}$. Tomemos $x_{0} \in M$ e consideremus a seguinte coleção $Z=$ $\left\{\overline{O^{+}(x)} ; x \in \overline{O^{+}\left(x_{0}\right)}\right\}$, cujos elementos são fechados, não vazios e invariantes de $M$. Além disso, o conjunto $Z$ é parcialmente ordenado, indutivo com respeito a inclusão de conjuntos; cada subconjunto linearmente ordenado de $Z$ tern um menor elemento, logo satisfaz as hipóteses do Lema de Zorn's. Então existe um elemento minimal $C_{x_{0}}=\overline{O^{+}\left(x_{1}\right)}$ para algum $x_{1} \in \overline{O^{+}\left(x_{0}\right)}$. Agora, como $C_{x_{0}}$ é minimal e $C_{x_{0}}=\overline{O^{+}(x)}, \forall x \in C_{x_{0}}$, portanto $C_{x_{0}}$ é conjunto controlável invariante.

Proposição 2.3.9 Se $M$ é compacto, então existe uma quantidade finita de conjuntos controláveis invariantes em $M$.

Demonstração: Vamos assumir que o seguinte conjunto $\left\{C_{i} ; i \in \mathbb{N}\right\}$ é uma coleção de conjuntos controláveis invariantes em $M$. Escolhemos $x_{i} \in C_{i}$ e como $M$ é compacto existe uma subseqüência $x_{i_{k}}$ tal que $\lim _{k \rightarrow \infty} x_{i_{k}}=x$. Tomemos agora $y \in \operatorname{int} C_{x}$, onde $C_{x}$ é definido na demonstração do Lema 2.3.8, então existem $t_{0} \in G$ e um controle $u_{0} \in \mathcal{U}$ com $y=\varphi\left(t_{0}, x, u_{0}\right)$. Agora, para uma vizinhança de $y, V_{y} \subset \operatorname{int} C_{x}$ existe uma vizinhança de $x, V_{x}$, tal que $\varphi\left(t_{0}, z, u_{0}\right) \in V_{y}, \forall z \in V_{x}$, mas a partir de um certo $n_{0} \in \mathbb{N}, i_{k}>n_{0}$, $x_{i_{k}} \in V_{x}, \operatorname{com} x_{i_{k}} \rightarrow C_{x}$, como $x_{i_{k}} \in C_{i_{k}} \Rightarrow C_{i_{k}}=C_{x}$. 
Proposição 2.3.10 Seja M uma variedade compactu. Entảo o sistema de controle (2.1) com int $O^{+}(x) \neq \emptyset$ e int $O^{-}(x) \neq \emptyset$ admite ao menos um conjunto controlável fechado e um aberto, e estes são exatamente os elementos maximal e minimal com respeito à ordem parcial.

\section{Demonstração:}

- Se $M$ é compacto, pelo Lema 2.3.8 existe ao menos um conjunto controlável invariante $C$ em $M$ e como por hipótese temos que int $O^{+}(x) \neq \emptyset$, então $C$ é fechado, e como $\overline{O^{+}(x)} \subset \bar{C}, \forall x \in C$, portanto conjuntos controláveis invariantes são elementos maximais de " $\leq "$.

- Consideremos agora o sistema reverso. Neste caso, int. $\widetilde{O}^{+}(x) \neq \emptyset$, onde $\widetilde{O}^{+}(x)$ é a órbita positiva do sistema reverso. Então existe um conjunto controlável invariante fechado do sistema reverso $B \subset M$.

Agora provemos que int $B$ é conjunto controlável aberto do sistema normal. De fato, como $B$ é conjunto controlável do sistema reverso, então dados $x, y \in \operatorname{int} B$, então $y \in \tilde{O}^{+}(x)$ pela Proposição 2.3.6, daí, $x \in O^{+}(y)$. Como isto é verdade, $\forall x, y$, então $\operatorname{int} B=O^{+}(x), \forall x \in \operatorname{int} B$. Isto prova que $\operatorname{int} B$ é conjunto pré-controlável para o sistema normal.

Resta mostrar que int $B$ satisfaz a maximalidade. Consideremos $C^{\prime}$ um conjunto controlável do sistema normal que contém $\operatorname{int} B, \operatorname{logo} C^{\prime}$ satisfaz (i) $\operatorname{int} C^{\prime} \neq \emptyset$, (ii) $C^{\prime} \subset \overline{O^{+}(x)}, \forall x \in C^{\prime}$ e (iii) $C^{\prime}$ é maximal. Seja $y \in C^{\prime}$ e $y \notin \operatorname{int} C^{\prime}$, de (ii) tem-se que $\operatorname{int} B \subset \overline{O^{+}(y)} \Rightarrow$ existe $x \in \operatorname{int} B$ tal que $x \in O^{+}(y) \Rightarrow y \in \tilde{O}^{+}(x)^{3}$, com $x \in \operatorname{int} B \Rightarrow y \in \operatorname{int} B$, absurdo! Logo int $B \subset C^{\prime} \Rightarrow C^{\prime}=\operatorname{int} B$.

\footnotetext{
${ }^{3} \widetilde{O}^{+}(x)$ é a órbita positiva do sistema reverso $\dot{x}=-X_{0}(x)-\sum_{i=1}^{m} u_{i}(t) X_{i}(x)$.
} 


\subsection{Núcleos de conjuntos controláveis}

Acontece que o interior de um conjunto controlável de um sistema em tempo discreto transitivo (acessível localmente) pode não ter as mesmas propriedades que o interior de conjunto controlável de um sistema em tempo contínuo, por esta razão o conceito de núcleo de um conjunto controlável $N(C)$ foi introduzido por Abertini-Sontag [1], que seria o "equivalente" ao interior de um conjunto controlável no caso contínuo, nesse caso. Mas definimos e provamos as propriedades do $N(C)$ tanto para o caso discreto como para o caso contínuo e na Seção 3.3 exibimos em particular um exemplo em tempo discreto, onde $N(C) \subset \operatorname{int} C$.

Definição 2.4.1 Seja $C \subset M$ um conjunto controlável com $\operatorname{int} C \neq \emptyset$, o núcleo de um conjunto controlável $C$ é definido como $N(C):=\left\{x \in \operatorname{int} C: \operatorname{int}\left(O^{+}(x) \cap C\right) \neq \emptyset e\right.$ $\left.\operatorname{int}\left(O^{-}(x) \cap C\right) \neq \emptyset\right\}$.

O resultado seguinte será útil para provar que $N(C)$ é aberto.

Proposição 2.4.1 Seja $A \subset M$ e definamos $A^{+}=\left\{x \in M: \operatorname{int}\left(O^{+}(x) \cap C\right) \neq \emptyset\right\}$. Então $A^{+}$é aberto.

Demonstração: Para provar o resultado devemos exibir a existência de um conjunto aberto inteiramente contido em $A^{+}$.

- Se $A^{+}=\emptyset$, nada temos que provar, pois $\emptyset$ é aberto.

- Suponhamos que $A^{+} \neq \emptyset$; seja $x \in A^{+}$. Como int $\left(O^{+}(x) \cap A\right) \neq \emptyset$, então existe um aberto $B \subset A$ (onde $B$ é o interior de alguma partição de $A$ ) tal que $B \subset O^{+}(x)$. logo existe $t \in G$ de maneira que $B \cap O_{t}^{+}(x)$ não tem medida nula; em particular, $O_{t}^{+}(x)$ não tem medida nula. Agora definimos $U_{B}^{t}=\left\{u: u \in U^{t}\right.$ e $\left.\varphi(t, x, u) \in B\right\}$ que é aberto, onde $U^{t}=\{\{u(i)\} \in U: i \in\{0, \ldots, t\}\}$ e $\varphi_{t, x}\left(U_{B}^{t}\right)$ não tem medida 
nula, aplicando o teorema de Sard's implica que $\frac{\partial \varphi}{\partial u}(t, x, u)$ é um isomorfismo, então existe $u \in U^{t}$ com posto $\frac{\partial \varphi}{\partial u}(f, x, u)=\operatorname{dim} M$. Podemos assumir sem perda de generalidade que $u \in \operatorname{int} U^{t}$. Por outro lado, seja uma vizinhança de $x, V_{x}$, tal que $\varphi\left(t, V_{x}, u\right) \subset B$ e ainda o posto $\frac{\partial \varphi}{\partial u}(t, y, u)=\operatorname{dim} \Lambda, \forall y \in V_{x} \Rightarrow$ pelo teorema do posto existc um aberto $V \subset A^{+}$.

Agora preparemos a prova da densidade do núcleo de $C$ com dois lemas.

Lema 2.4.2 Consideremos um conjunto controlável $C \subset M$, com $\operatorname{int} C \neq \emptyset$, se $N(C) \neq \emptyset$. Então $C \subset \tilde{O}^{+}(x)$, para qualquer $x \in N(C)$. onde $\tilde{O}^{+}(x)$ é a órbita positiva do sistema reverso.

Demonstração: Seja $x \in N(C)$ e $y \in C, \Rightarrow \operatorname{int} \tilde{O}^{+}(x) \cap C \neq \emptyset$. Como $C$ é conjunto controlável, $\Rightarrow O^{+}(y) \cap \operatorname{int} \tilde{O}^{+}(x) \neq \emptyset \Rightarrow y \in \operatorname{int}, \tilde{O}^{+}(x)$. Portanto, $C \subset \operatorname{int} \tilde{O}^{+}(x)$.

Lema 2.4.3 Seja um conjunto controlável $C \subset M$, com int $C \neq \emptyset$, se $N(C) \neq \emptyset$, para qualquer $x \in N(C)$ tem-se $N(C)=\operatorname{int} O^{+}(x) \cap \operatorname{int} O^{-}(x)$.

Demonstração: Seja $y \in \operatorname{int} O^{+}(x) \cap \operatorname{int} O^{-}(x)$

$$
\begin{aligned}
& \Rightarrow y \in \operatorname{int} O^{+}(x) \quad \text { e } \quad y \in \operatorname{int} O^{-}(x) \\
& \Rightarrow x \in \operatorname{int} \tilde{O}^{+}(y) \quad \text { e } \quad x \in \operatorname{int} O^{+}(y) .
\end{aligned}
$$

Como int $\tilde{O}^{+}(y)$ e $\operatorname{int} O^{+}(y)$ são densos em $\tilde{O}^{+}(y)$ e $O^{+}(y)$, respectivamente,

$$
\begin{aligned}
& \Rightarrow \operatorname{int} O^{+}(y) \cap \operatorname{int} C \neq \emptyset \text { e } \operatorname{int} \widetilde{O}^{+}(y) \cap \operatorname{int} C \neq \emptyset \\
& \Rightarrow y \in N(C) .
\end{aligned}
$$

Para provar a recíproca, seja $x, y \in N(C)$. Pelo Lema 2.4.2 temos $y \in \operatorname{int} O^{-}(x)$ e $x \in \operatorname{int} O^{-}(y)\left(y \in \operatorname{int} O^{+}(x)\right)$, isto é, $y \in \operatorname{int} O^{+}(x) \cap \operatorname{int} O^{-}(x)$. 
Nos próximos dois resultados, nos quais temos controlabilidade exata. o subconjunto $N(C) \subset C$ é aberto e denso em $C \subset M$.

Proposição 2.4.4 Consideremos $C \subset M$ um conjunto controlável com int $C \neq \emptyset$ para 0 sistema (2.1) com $N(C) \neq \emptyset$. Então o núcleo de um conjunto controlável $N(C)$ é denso em $C$.

Demonstração: Vamos provar que $C \subset \overline{N(C)}$. Seja $x \in N(C)$, pelo Lema 2.4 .3 e pelo fato que int $O^{+}(x) \neq \emptyset$ e int $O^{-}(x) \neq \emptyset$ são abertos, temos que $\overline{N(C)}=\overline{\operatorname{int} O^{+}(x)} \cap$ $\operatorname{int} \widetilde{O}^{+}(x)$. Logo, basta provar que $\dot{C} \subset \overline{\operatorname{int} O^{+}(x)}$ e $C \subset \operatorname{int} \tilde{O}^{+}(x)$.

De fato, como $O^{+}(x) \subset O^{+}(\varphi(t, x, u)) \subset \operatorname{int} O^{+}(x) \Rightarrow \overline{O^{+}(x)} \subset \overline{\operatorname{int} O^{+}(x)}, \operatorname{logo} C \subset$ $\overline{O^{+}(x)} \subset \overline{\operatorname{int} O^{+}(x)}$ e $C \subset \operatorname{int} \tilde{O}^{+}(x)\left(\right.$ Lema 2.4.2). Portanto, $C \subset \overline{\operatorname{int} O^{+}(x)} \cap \operatorname{int}_{O^{+}}(x)=$ $\overline{N(C)}$.

Proposição 2.4.5 Seja $C \subset M$ um conjunto controlável com int $C \neq \emptyset$ para (2.1). Então o núcleo de $C$ é aberto.

Demonstração: Como pela Proposição $2.4 .1 \operatorname{int}\left(O^{+}(x) \cap C\right) \neq \emptyset \operatorname{eint}\left(\tilde{O}^{+}(x) \cap C\right) \neq \emptyset$ são abertos, então $N(C)$ é aberto. 


\section{Capítulo 3}

\section{O sistema dinâmico associado ao sistema de controle}

No contexto de sistemas dinâmicos, fenômenos com dinâmica complicada ocorrem quando pequenas mudanças nas condições iniciais causam grandes mudanças na evolução do sistema. Este tipo de dinâmica é chamada caótica, cuja teoria matemática tem ocupado, principalemente nos últimos vinte anos, a atenção de matemáticos e cientistas de outras áreas e vem experimentando um desenvolvimento marcante. Mas a teoria de controle é mais antiga, com aplicações em diversos campos da ciência. Neste capítulo estudamos como certas extensões que são invariantes $\mathcal{C} \subset \mathcal{U} \times M$ de um conjunto controlável $D \subset M$ satisfazem as propriedades de caos, onde $\mathcal{U} \times M$ é um espaço topológico. Assim, mostramos que sistemas de controle são caóticos em seus conjuntos controláveis.

Definição 3.0.1 No espaço topológico $\mathcal{U} \times M$ a função $\Psi: G \times \mathcal{U} \times M \rightarrow \mathcal{U} \times M$ definida por $\Psi(t, u, x)=(h(t, u(\cdot)), \varphi(t, x, u))$ será chamado fluxo controlável associado ao sistema de controle (2.1), onde h é o shift discutido na Seção 1.4 e $\varphi(t, x, u$ ) é a solução de (2.1) correspondente a u e x.

Esta função está bem definida, ist.o é, $\Psi(t, u, x) \in \mathcal{U} \times M$, pois $h_{t}(u(\cdot))=u(\cdot+t) \in \mathcal{U}$ e $\varphi(t, \cdot, u): M \rightarrow M$ tal que $\varphi(t, x, u)=y \in M, \operatorname{logo}\left(h_{t} u, y\right) \in \mathcal{U} \times M$. 
Proposição 3.0.1 O fluxo controlável $\Psi$ define um sistema dinâmico contínuo em $\mathcal{U} \times M$.

\section{Demonstração:}

- $\Psi$ satisfaz as propriedades de grupo. De fato:

$$
\begin{aligned}
\Psi_{t+s}(u, x) & =\left(h_{t+s} u, \varphi(t+s, x, u)\right) \\
& =\left(h_{t} \cdot h_{s} u, \varphi\left(t, \varphi(s, x, u), u_{s}(\cdot)\right)\right. \\
& =\left(h_{t} u^{\prime}, \varphi\left(t, x^{\prime}, h_{s} u\right)\right)=\left(h_{t} u^{\prime}, \varphi\left(t, x^{\prime}, u^{\prime}\right)\right) \\
& =\Psi_{t}\left(u^{\prime}, x^{\prime}\right) \\
& =\Psi_{t}\left(\Psi_{s}(u, x)\right) .
\end{aligned}
$$

Portanto,

$$
\Psi_{t+s}=\Psi_{t} \circ \Psi_{s} \quad \text { e } \quad \Psi_{u, x}(0)=(u, x) .
$$

- $\Psi$ é contínua se cada coordenada é contínua; mas, pelo Lema 1.4 .3 , o shift $h$ é contínuo. Então resta mostrar que $\varphi(t, x, u)$ é contínuo e para isso vamos usar o Lema de Gronwall.

De fato, sejam $\left\{x_{n}\right\}_{n \in \mathbb{Z}^{+}}$e $\left\{u_{n}\right\}_{n \in \mathbb{Z}^{+}}$seqüências em $M$ e $\mathcal{U}$, respectivamente, tais que $x_{n} \rightarrow x_{0}$ e $u_{n} \rightarrow v$, vamos definir $\varphi_{n}(t):=\varphi\left(t, x_{n}, u_{n}\right), \varphi_{0}(t):=\varphi\left(t, x_{0}, v\right)$ e mostrar que a seqüência $\varphi\left(t, x_{n}, u_{n}\right) \rightarrow \varphi\left(t, x_{0}, v\right), \forall t \in G$. Isto segue da seguinte estimativa e serem os campos de vetores globalmente Lipschitziana.

$$
\begin{aligned}
\left|\varphi_{n}(t)-\varphi_{0}(t)\right| \leq & \left|x_{n}-x_{0}\right|+\mid \int_{0}^{t}\left\{X_{0}\left(\varphi_{n}(t)\right)+\sum_{i=1}^{m} u_{n, i}(t) X_{i}\left(\varphi_{n}(t)\right)\right. \\
& \left.-X_{0}\left(\varphi_{0}(t)\right)-\sum_{i=1}^{m} v_{i}(t) X_{i}\left(\varphi_{0}(t)\right)\right\} \mathrm{d} t \mid \\
\leq & \left|x_{n}-x_{0}\right|+\mid \int_{0}^{t}\left\{X_{0}\left(\varphi_{n}(t)\right)-X_{0}\left(\varphi_{0}(t)\right)\right. \\
& \left.+\sum_{i=1}^{m} u_{n, i}(t)\left[X_{i}\left(\varphi_{n}(t)\right)-X_{i}\left(\varphi_{0}(t)\right)\right]\right\} \mathrm{d} t \mid
\end{aligned}
$$




$$
+\left|\int_{0}^{t}\left\{\sum_{i=1}^{m}\left[u_{n, i}(t)-v_{i}(t)\right] X_{i}\left(\varphi_{0}(t)\right)\right\} \mathrm{d} t\right|
$$

é imediata pela assunção que o primeiro e terceiro somando converge a zero, fica então

$$
\left|\varphi_{n}(t)-\varphi_{0}(t)\right| \leq 0+c_{1} \int_{0}^{t}\left|\varphi_{n}(t)-\varphi_{0}(t)\right| \mathrm{d} t
$$

onde $c_{1}$ é uma constante de Lipschitz. Para os $(m+1)$ campos de vetores.

Aplicamos o Lema de Gronwall quando $\alpha=0$ e temos $\left|\varphi_{n}(t)-\varphi_{0}(t)\right| \rightarrow 0$. Portanto, $\varphi\left(t, x_{n}, u_{n}\right) \rightarrow \varphi\left(t, x_{0} \cdot v\right)$.

\subsection{Caos na extensão dos conjuntos controláveis}

Aqui apresentamos os conceitos que interessam neste capítulo e que foram introduzidos por F. Colonius e W. Kliemann [4].

Definição 3.1.1 Seja $C \subset M$ um conjunto controlável de (2.1), dizemos que

$$
\mathcal{C}=\overline{\{(u, x) \in \mathcal{U} \times M, \varphi(t, x, u) \in \operatorname{int} C, \forall t \in G\}}
$$

é a extensão do conjunto controlável $C \subset M \operatorname{com}$ int $C \neq \emptyset$, onde $\varphi$ é solução de (2.1).

OBS:

- O fecho é tomado com respeito à topologia produto $w^{*}$ (da convergência) em $\mathcal{U}\left(U^{\mathbb{Z}}\right)$ e a topologia dada em $M$.

- Se $C$ é limitado em alguma métrica dada em $M$, então $\mathcal{C}$ é compacto.

Lema 3.1.1 $\mathcal{C}$ é um conjunto $\Psi$-invariante.

Demonstração: Seja $(u, x) \in \mathcal{C}$, queremos mostrar que $\Psi_{t}(u, x)=\left(h_{t} u, \varphi(t, x, u)\right) \in \mathcal{C}$.

De fato,

$$
\begin{aligned}
\Psi_{t}(u, x) & =\left(h_{t} u, \varphi(t, x, u)\right)=\varphi\left(s, h_{t} u, \varphi(t, x, u)\right) \in \operatorname{int} C \\
& \Rightarrow \varphi(s+t, u, x) \in \operatorname{int} C \Rightarrow \Psi_{t}(u, x) \in \mathcal{C}
\end{aligned}
$$


Lema 3.1.2 Seja $C \subset M$ um conjunto controlável para (2.1). com $\operatorname{int} C \neq \emptyset$ e $\subset \mathcal{U} \times M$ definido em 3.1.1. Então os pontos periódicos de $\Psi$ sào densos em $\mathcal{C}$.

Demonstração: Vamos mostrar que cada ponto da forma $(u, x) \in \mathcal{C} \operatorname{com} \varphi(t, x, u) \in$ int $C, \forall t \in G$, pode ser aproximado por pontos periódicos.

Escolhemos $(u, x) \in \mathcal{C}$ e tomemos uma vizinhança de $(x, u)$ da forma $\mathcal{W}=(\mathcal{V} \times \mathcal{N}) \cap \mathcal{C}$. onde $\mathcal{N} \subset M, \mathcal{V} \subset \mathcal{U}$. Podemos assumir que $\mathcal{V}$ tem a forma

$$
\mathcal{V}=\left\{v \in \mathcal{U},\left|\int_{\mathbb{R}}\left\langle u(t)-v(t), f_{i}(t)\right\rangle \mathrm{d} t\right|<\epsilon, \text { para } i=1, \ldots k\right\},
$$

onde $f_{i} \in L^{1}\left(\mathbb{R}, \mathbb{R}^{m}\right)$. Então existem $T>0$ e $\epsilon_{0}=\frac{\epsilon}{\operatorname{diam} U}>0$ tais que $\int_{\mathbb{R} \backslash \backslash-T, T]}\left|f_{i}(t)\right| \mathrm{d} t<$ $\epsilon_{0}$. Como $(u, x) \in \mathcal{C}, \varphi(-T, x, u)$ e $\varphi(T, x, u)$ pertencem a int $C$, logo, por controlabilidade exata em int $C$, existem $T_{1}>0$ e $u^{0} \in \mathcal{U}$ tais que $\varphi\left(T_{1}, \varphi(T, x, u), u^{0}\right)=\varphi(-T, x, u)$. No que segue vamos definir $u_{p} \in \mathcal{U}$ da seguinte maneira:

$$
u_{p}(t)= \begin{cases}u(t) & ,-T \leq t \leq T \\ u^{0}(t-T) & , T<t<T+T_{1}\end{cases}
$$

e estendamos $u_{p}$ a uma função de período $\left(2 T+T_{1}\right)$ em $G$. A trajetória correspondente $\varphi\left(\cdot, x, u_{p}\right)$ também é de período $\left(2 T+T_{1}\right)$. Agora, aplicando a maximalidade de conjuntos controláveis temos $\varphi\left(\cdot, x, u_{p}\right) \in \operatorname{int} C$ e assim $\left(u_{p}, x\right)$ é um ponto periódico de $\Psi$ em $\mathcal{U} \times M$. Além disso,

$$
\left|\int_{\mathbb{R}}\left\langle u(t)-u_{p}(t), f_{i}(t)\right\rangle \mathrm{d} t\right| \leq \operatorname{diam} U \int_{\mathbb{R} \backslash[-T, T]}\left|f_{i}(t)\right| \mathrm{d} t<\epsilon, \quad \forall i=1, \ldots, k,
$$

isto é, $u_{p} \in \mathcal{V}$, portanto $\left(u_{p}, x\right) \in \mathcal{W}$.

Lema 3.1.3 Consideremos $C \subset M$ um conjunto controlável para (2.1) com int $C \neq \emptyset e$ $\mathcal{C} \subset \mathcal{U} \times M$ definido em 3.1.1. Entâo o sistema dinâmico $(\mathcal{C}, G, \Psi)$ é topologicamente mixing e transitivo.

Demonstração: Sejam os conjuntos abertos não vazios $\mathcal{W}_{1}, \mathcal{W}_{2}$ de $\mathcal{C}$, temos que demonstrar que existem $T_{1}>0, T_{0}, T_{1} \in G$ tais que $\mathcal{W}_{1} \cap \Psi\left(-n T_{1}+T_{0}, \mathcal{W}_{2}\right) \neq \emptyset, \forall n \in \mathbb{N}$, isto é, existem um ponto $p$ em $\mathcal{W}_{1}$ e um número natural $n$ tais que $\Psi^{n}(p)$ está em $\mathcal{W}_{2}$. 
Pelo Lema 3.1.2. os pontos periódicos de $\Psi$ são densos em $\mathcal{C}$. logo existe $\left(u_{j} . x_{j}\right) \in \mathcal{W}_{j}$ tais que $\left(u_{j}, \varphi\left(\cdot, x_{j} \cdot u_{j}\right)\right)$ é $t_{j}$-periódico para algum $t_{j}>0, j=1$. 2. Daí bastará tomar $\mathcal{W}_{j}$ da forma $\mathcal{W}_{j}=\left(\mathcal{V}_{j} \times \mathcal{N}_{j}\right) \cap \mathcal{C} \operatorname{com} \varphi\left(t, x_{j}, u_{j}\right) \in \operatorname{int} C, \forall t \in G$.

$$
\begin{aligned}
\mathcal{V}_{j} & =\left\{v \in \mathcal{U},\left|\int_{\mathbb{R}}\left\langle u_{j}(\tau)-v(\tau), g_{i j}(\tau)\right\rangle d \tau\right|<\epsilon, \quad \text { para } j=1,2, \quad\right. \text { e } \\
\mathcal{N}_{j} & =\left\{y \in M, d\left(x_{j}, y\right)<\epsilon\right\},
\end{aligned}
$$

onde $d$ representa a distância em $M$. Então existem $T>0$ e $\epsilon_{0}=\frac{\epsilon}{\operatorname{diam} U}>0$ tais que $\int_{\mathbb{R} \backslash\{-T, T \mid} \mid g_{i j}(\tau) d \tau<\epsilon_{0}$, para $j=1,2, i=1,2, \ldots, k_{j}$. Vamos assumir que $t_{1}, t_{2}>T$, além disso, $\varphi\left(t, x_{j}, u_{j}\right) \in \operatorname{int} C, \forall t \in G$. Então existem $t_{0}>0, v_{0} \in \mathcal{U} \operatorname{com} \varphi\left(t_{0}, x_{2}, v_{0}\right)=x_{1}$.

Seja $n \in \mathbb{N}$ fixo e definamos $v_{n} \in \mathcal{U}$ pela fórmula

$$
v_{n}(\tau)= \begin{cases}u_{1}(\tau) & ,-n t_{1} \leq \tau<\infty \\ v_{0}\left(\tau+n t_{1}+t_{0}\right) & ,-n t_{1}-t_{0} \leq \tau<-n t_{1}, \\ u_{2}\left(\tau+n t_{1}+t_{0}\right) & ,-\infty<\tau<-n t_{1}-t_{0}\end{cases}
$$

então $v_{n} \in \mathcal{V}_{1}$, pois

$$
\left|\int_{\mathbb{R}}\left\langle u_{1}(\tau)-v_{n}(\tau), g_{i 1}(\tau)\right\rangle d \tau\right| \leq \operatorname{diam} U \int_{\mathbb{R} \backslash\left[-T_{1}, T_{2}\right]}\left|g_{i 1}(\tau)\right| d \tau<\epsilon
$$

Além disso,

$$
\begin{aligned}
& \varphi\left(t, x_{1}, v_{n}\right)=\varphi\left(t, x_{1}, u_{1}\right), \quad \text { para } t \geq-n t_{1} \mathrm{e} \\
& \varphi\left(t, x_{1}, v_{n}\right)=\varphi\left(t+n t_{1}+t_{0}, x_{2}, u_{2}\right) \text { para } t<-n t_{1}-t_{0} .
\end{aligned}
$$

Portanto, $\varphi\left(t, x_{1}, v_{n}\right) \in \operatorname{int} C, \forall t \in G$. Isso mostra que $\left(v_{n}, x_{1}\right) \in \mathcal{W}_{1}$.

Por outro lado, temos

$$
\begin{aligned}
& \varphi\left(-n t_{1}-t_{0}-t_{2}, x_{1}, v_{n}\right)=x_{2} \quad \mathrm{e} \\
& v_{n}\left(-n t_{1}-t_{0}-t_{2}+\tau\right)=u_{2}\left(\tau-t_{2}\right)=u_{2}(\tau) \text { para } \tau<t_{2}
\end{aligned}
$$

Portanto,

$$
\left|\int_{\mathbb{R}}\left\langle u_{2}(\tau)-v_{n}\left(-n t_{1}-t_{0}-t_{2}+\tau\right), g_{i 2}(\tau)\right\rangle d \tau\right|
$$




$$
\begin{aligned}
& =\left|\int_{t_{2}}^{\infty}\left\langle u_{2}(\tau)-v_{n}\left(-n t_{1}-t_{0}-t_{2}+\tau\right), g_{i 2}(\tau)\right\rangle d \tau\right| \\
& \leq \operatorname{diam} U \int_{t_{2}}^{\infty}\left|g_{i 2}(\tau)\right| d \tau \\
& <\epsilon, \quad \forall i=1 \ldots k_{2} .
\end{aligned}
$$

Finalmente,

$$
\begin{aligned}
\Psi\left(-n t_{1}-\left(t_{0}+t_{2}\right), v_{n}, x_{1}\right) & =\left[v_{n}\left(-n t_{1}-\left(t_{0}+t_{2}\right)+\cdot\right), \varphi\left(-n t_{1}-\left(t_{0}+t_{2}\right), x_{1}, v_{n}\right)\right] \\
& =\left[h\left(-n t_{1}-\left(t_{0}+t_{2}\right), v_{n}\right), x_{2}\right] \in \mathcal{W}_{2} \\
\text { se } T_{1}=t_{1} \quad \text { e } T_{0}=-t_{0}-t_{2} & =\left[h\left(-n T_{1}+T_{0}, v_{n}\right), x_{2}\right] \in \mathcal{W}_{2} .
\end{aligned}
$$

Mañe, R. [11] mostrou que aplicações contínuas em espaços métricos de Baire transitividade é implicado de mixing.

Lema 3.1.4 Suponhamos que $C \subset M$ é um conjunto controlável para (2.1) com $\operatorname{int} C \neq \emptyset$ $e \mathcal{C} \subset \mathcal{U} \times M$ definido em 3.1.1. Então o sistema dinâmico $(\mathcal{C}, G, \Psi)$ tem dependência sensitiva em condiçôes iniciais.

Demonstração: A fim de demonstrar o resultado, precisamos de uma métrica em $\mathcal{U} \times M$. Seja $d_{p}$ qualquer $p$-métrica $(p \in(0, \infty])$ no espaço produto com respeito a $d_{u}, d_{M}$, onde $d_{u}$ é a métrica definida em (1.1) e $d_{M}$ é qualquer métrica em $M$, mostramos no Lema 1.4.5 que o shift tem dependência sensitiva, isto é, $d_{\mathcal{U}}(h(t, u), h(t, v)) \geq \delta$. Então, como conseqüência imediata, temos que

$$
d_{p}(\Psi(t, u, x), \Psi(t, v, y)) \geq \delta
$$

Mostramos que o fluxo $\Psi: \mathcal{U} \times M \rightarrow \mathcal{U} \times M$, restrito à sua extensão $\mathcal{C}$, goza das mesmas propriedades do fluxo shift, isto é, são caóticos. 


\subsection{Controle de caos}

Se $\Psi$ não é transitivo em seu domínio. então será que existem regiões onde podemos controlar pelo menos aproximadamente? As duas proposições seguintes respondem parcialmente a questão.

Proposição 3.2.1 Seja $(u, x) \in \mathcal{U} \times M$ um ponto periódico de $\Psi$. Então existe um conjunto controlável $C \subset M$ tal que $x \in C$.

Demonstração: Como $(u, x)$ é um ponto $\Psi$-periódico, então a trajetória $\varphi(\cdot, x, u)$ de (2.1) é uma órbita periódica, $\forall t>0$. Logo, existe um conjunto pré-controlável $C^{\prime}$ e, portanto, pelo Lema 2.2.8 existe um único conjunto controlável $C$ tal que $C^{\prime} \subset C$.

Proposição 3.2.2 Vamos considerar um conjunto invariante e fechado $\mathcal{W} \subset \mathcal{U} \times M$, tal que o sistema dinâmico $(\mathcal{W}, G, \Psi)$ é topologicamente mixing. Então existe um conjunto controlável $C \subset M$, com $\pi_{M} \mathcal{W}=\{x \in M$, existe $u \in \mathcal{U}$ com $(u, x) \in \mathcal{W}\} \subset C$, onde $\pi_{M}: \mathcal{U} \times M \rightarrow M$ é a projeção na segunda componente.

Demonstração: Como $\mathcal{W}$ é topologicamente mixing, então $\forall \epsilon>0$, existe $t_{k}>0$ arbitrariamente grande, com $d\left(\varphi\left(t_{k}, x, u\right), y\right)<\epsilon \Rightarrow \forall x, y \in \pi_{M} \mathcal{W}$, existe $u \in \mathcal{U}$ tal que $\varphi\left(t_{k}, x, u\right) \rightarrow y$ para uma seqüência $t_{k} \rightarrow \infty \Rightarrow$ existe um conjunto pre-controlável $C^{\prime}$. Novamente, pelo Lema 2.2.8, existe um único controlável $C$.

O resultado que segue mostra a relação entre os conceitos do fluxo de controle $(\mathcal{U} \times M$, $G, \Psi)$ e os conjuntos controláveis de (2.1).

Teorema 3.2.3 Seja o sistema de controle (2.1) com a propriedade de acessibilidade local. 
(i) Se $C \subset M$ é um conjunto controlável com $\operatorname{int} C \neq \emptyset$. então $\mathcal{C}$ definido em 3.1.1 é um conjunto topologicamente transitivo (ou mixing) maximal, com

$$
\text { (a) } \overline{\pi_{M} \mathcal{C}}=\bar{C} \quad e \quad \text { (b) } \operatorname{int} \pi_{M} \mathcal{C}=\operatorname{int} C
$$

onde $\pi_{n} \mathcal{C}=\{x \in M$. existe $u \in \mathcal{U}$ tal que $(u, x) \in \mathcal{C}\}$.

(ii) $S e \mathcal{C} \subset \mathcal{U} \times M$ é um conjunto topologicamente transitivo (ou mixing) maximal de $(\mathcal{U} \times M, G, \Psi)$, com int $\pi_{M} \mathcal{C} \neq \emptyset$, então existe um conjunto controlável que satisfaz (a) $e(b)$.

\section{Demonstração:}

$(\Rightarrow)$ Provamos no Lema 3.1.3 que $\mathcal{C}$ é topologicamente mixing, portanto transitivo.

- Para a afirmativa (a) lembramos que o fecho de $\mathcal{C}$ implica o fecho de $\pi_{M} \mathcal{C}$.

- Vamos provar (b).

Como provamos no Capítulo 2, Lema 2.2.4, que $\bar{C}=\overline{\operatorname{int} C}$ e pela prova no Lema 3.1.2, temos que para cada $x \in \operatorname{int} C$ existe $u \in \mathcal{U}$ tal que $\varphi(\cdot, x, u)$ é periódico e $\varphi(\cdot, x, u) \in \operatorname{int} C$.

- Falta apenas provar a maximalidade de $\mathcal{C}$.

Se $\mathcal{C}^{\prime} \supset \mathcal{C}$ é topologicamente transitivo, então existe $(u, x) \in \mathcal{C}^{\prime}$ tal que $w(u, x)=\mathcal{C}^{\prime}$, onde $w(\cdot, \cdot)$ é o $w$-limite; então existe $t_{0} \in \mathbb{R}$ tal que $\varphi(t, x, u) \in$ $\operatorname{int} C, \forall t \geq t_{0}$. Como $y:=\varphi\left(t_{0}, x, u\right) \in \operatorname{int} C$, então existe $v \in \mathcal{U}$ com $\varphi(t, y, v) \in \operatorname{int} C, \forall t<0$. Seja $u^{0} \in \mathcal{U}$ definido da forma

$$
u^{0}(t)= \begin{cases}u(t) & , t \geq t_{0} \\ v\left(t+t_{0}\right) & , t<t_{0}\end{cases}
$$

então $\left(u^{0}, y\right) \in \mathcal{C}, \varphi\left(t, y, u^{0}\right) \in \operatorname{int} C, \forall t \in \mathbb{R}$, e $w\left(u^{0}, y\right)=w(u, x)=\mathcal{C}^{\prime}$. Logo, como $\mathcal{C}$ é fechado, temos $\mathcal{C}^{\prime}=w\left(u^{0}, y\right) \subset \mathcal{C}$, portanto $\mathcal{C}$ é maximal. 
$(\Leftarrow)$ Tomemos um conjunto $\mathcal{C}$ topologicamente transitivo maximal com $\operatorname{int} \pi_{M l} \mathcal{C} \neq \emptyset$. Vamos mostrar que para $x_{1}, x_{2} \in \operatorname{int} \pi_{M} \mathcal{C}$ existe um controle $u \in \mathcal{U}$ e tempo $t>0$ $\operatorname{com} \varphi\left(t, x_{1}, u\right)=x_{2}$.

Seja $y_{1} \in \operatorname{int} O^{+}\left(x_{1}\right) \cap \operatorname{int} \pi_{M} \mathcal{C}$ e $y_{2} \in \operatorname{int} O^{-}\left(x_{2}\right) \cap \operatorname{int} \pi_{M} \mathcal{C}$.

Agora consideremos vizinhanças de $y_{1}, V_{y_{1}}^{1}$, e de $y_{2}, V_{y_{2}}^{2}$, com a seguinte condição: $V^{1} \subset \operatorname{int} O^{+}\left(x_{1}\right)$ e $V^{2} \subset \operatorname{int} O^{-}\left(x_{2}\right)$. Como $\mathcal{C}$ é topologicamente transitivo, então existe $(u, x) \in \mathcal{C}$ tal que $w(u, x)=\mathcal{C}$. Então um ponto em $V^{2}$ pode ser atingido de um ponto em $V^{1}$, portanto $x_{2}$ pode ser atingido de $x_{1}$ então existe um conjunto controlável $C \subset M \operatorname{com} \operatorname{int} \pi_{M} \mathcal{C} \subset \operatorname{int} C$. Agora $C$ pode ser estendido a um conjunto $\tilde{\mathcal{C}}$ topologicamente maximal mixing, como na definição.

Afirmação: $\mathcal{C} \supset \tilde{\mathcal{C}}$; então a maximalidadt de $\tilde{\mathcal{C}}$ prova que $\mathcal{C}=\tilde{\mathcal{C}}$, como queríamos. Como $w(u, x)=\mathcal{C}$ e int $\pi_{M} \mathcal{C} \subset \operatorname{int} C=\operatorname{int} \pi_{M} \tilde{\mathcal{C}}$, então $\left(u^{0}, y\right) \in \tilde{\mathcal{C}} \operatorname{com} w\left(u^{0}, y\right)=\mathcal{C}$. Agora a invariânça de $\tilde{\mathcal{C}}$ implica que $\tilde{\mathcal{C}} \subset \mathcal{C}$.

Corolário 3.2.4 Se vale a propriedade de acessibilidade local para (2.1), então as seguintes afirmações sâo equivalentes:

(i) o sistema dinâmico $(\mathcal{U} \times M, G, \Psi)$ é topologicamente transitivo;

(ii) o sistema dinâmico $(\mathcal{U} \times M, G, \Psi)$ é topologicamente mixing;

(iii) os sistema de controle (2.1) é completamente controlável em $M$.

\subsection{Especificidades do caso discreto}

A relação entre os conjuntos controláveis de um sistema de controle e o comportamento caótico em subconjuntos de um sistema dinâmico de dimensão infinita demonstrado neste capítulo para o caso de tempo contínuo, tem sua extensão ao caso de tempo discreto que foi estudado em detalhe por Albertini-Sontag [1], mas existe uma dificuldade que ainda sob 
a assunção de acessibilidade local no interior de conjuntos controláveis no tempo discreto não é válida a controlabilidade exata. Então eles introduzem o conceito de núcleo de um conjunto controlável. discutido no Capítulo 2. seção 2.4, onde mostramos que $N(C)$ é um subconjunto de $C$, aberto e denso em $C$, no qual temos a controlabilidade exata.

O exemplo que segue é para mostrar que no caso do tempo discreto a fórmula (b) do Teorema 3.2.3 sempre é uma inclusão e não uma igualdade como no caso contínuo, além disso, $N(C) \subset \operatorname{int} C$.

Exemplo [1] Vamos considerar o seguinte sistema analítico em tempo discreto: seja a variedade $M=\mathbb{R}^{2}, U=[-1,1] \times[-1,1]$, e a aplicação $f_{u, v}: \mathbb{R}^{2} \rightarrow \mathbb{R}^{2}$ definido por $f_{u, v}(x, y)=f(x, y, u, v)=\left(x+1+u y ; y+\frac{v}{2} g(x)\right)$, onde $g: \mathbb{R} \rightarrow \mathbb{R}$ é definido por $g(x)=\frac{\operatorname{sen}(\pi x)}{\pi x}$. Podemos verificar que $\left|g^{\prime}(x)\right| \leq 1, \forall x \in \mathbb{R}$.

De fato,

$$
f(y)=\frac{\operatorname{sen} y}{y}, \quad y \neq 0, \quad f^{\prime}(y)=\frac{y \cos y-\operatorname{sen} y}{y^{2}} .
$$

Vamos mostrar que $-1 \leq \frac{y \cos y-\operatorname{sen} y}{y^{2}} \leq 1$ ou, equivalentemente, $-y^{2} \leq y \cos y-\operatorname{sen} y \leq y^{2}$. Fazendo $h(y)=y^{2}-y \cos y+\operatorname{sen} y, \operatorname{temos} h(0)=0$ e $h^{\prime}(y)=2 y-\cos y+y \operatorname{sen} y+\cos y=$ $y(2+\operatorname{sen} y)$.

$$
\begin{aligned}
& h^{\prime}(y)>0 \text { se } y>0 \Rightarrow h \text { crescente para } y>0, \\
& h^{\prime}(y)<0 \text { se } y<0 \Rightarrow h \text { decrescente para } y<0 .
\end{aligned}
$$

Portanto $h(y) \geq 0 \Rightarrow y \cos y-\operatorname{sen} y \leq y^{2}$.

Analogamente, temos o outro caso. Fazendo $k(y)=y \cos y-\operatorname{sen} y+y^{2}$, temos $k(0)=0$ e $k^{\prime}(y)=\cos y-y \operatorname{sen} y-\cos y+2 y=y(2-\operatorname{sen} y)$.

$$
\begin{aligned}
& k^{\prime}(y)>0 \text { se } y>0, \\
& k^{\prime}(y)<0 \text { se } y<0 .
\end{aligned}
$$

Portanto $k(y) \geq 0 \Rightarrow y \cos y-\operatorname{sen} y \geq-y^{2}$, e $g(x)=0$ quando $x \in \mathbb{Z} \backslash\{0\}$.

Vamos verificar também que $f_{u, v}(\cdot, \cdot)$ é invertível e bijetora, $\forall(u, v) \in U$. 
- $J f_{u, v}(x, y)=\left[\begin{array}{ll}\frac{\partial f_{1}}{\partial x} & \frac{\partial f_{1}}{\partial y} \\ \frac{\partial f_{2}}{\partial x} & \frac{\partial f_{2}}{\partial y}\end{array}\right]=\left[\begin{array}{cc}1 & u \\ \frac{v}{2} g^{\prime}(x) & 1\end{array}\right] . \quad$ Logo det $J f_{u, v}(x, y)=1-\frac{u v}{2} g^{\prime}(x)$ Como $u, v \in[-1.1]$ e $\left|g^{\prime}(x)\right| \leq 1$, portanto $\left|\frac{u v}{2} g^{\prime}(x)\right| \leq \frac{1}{2}$. Ent.ão det $J f_{u, v}(x, y) \neq 0$. $\forall x, y \in M$.

- $f_{u, v}(x, y)=\left(x+1+u y, y+\frac{v}{2} g\left(x_{\prime}^{\prime}\right)=(a, b)\right.$

$$
\left\{\begin{array}{l}
x+1+u y=a \\
\frac{v}{2} g(x)+y=b
\end{array}\right.
$$

Multiplicando a segunda equação por $(-u)$ temos $x+1-\frac{u v}{2} g(x)=a-u b$ e fazendo $h(x)=x+1-\frac{u v}{2} g(x)-a+u b=0$. Então vemos que

$$
\lim _{x \rightarrow-\infty} h(x)=-\infty \quad \text { e } \quad \lim _{x \rightarrow+\infty}=+\infty
$$

Logo temos a sobrejeção. Derivando tem-se $h^{\prime}(x)=1-\frac{u v}{2} g^{\prime}(x), h^{\prime}(x) \geq 0$. Então $f_{u, v}(\cdot, \cdot)$ é injetora, portanto bijetora.

Para este sistema podemos ver que para todo $t \in \mathbb{N}$ com $t \geq 1$ vale o seguinte:

1. os pontos do tipo $(-t, 0)$ não são acessíveis no passado;

2. os pontos do tipo $(t, 0)$ não são acessíveis no futuro.

Seja $C_{1}=\{(t, 0): t \in \mathbb{N}, t \geq 1\}$, queremos mostrar que $C=M \backslash C_{1}$ é um conjunto controlável.

- Observe que $C$ é certamente maximal. De fato, pontos que não estão em $C_{1}$ deveriam pertencer a um conjunto controlável, pois não são acessíveis no futuro.

- Para provar que $C$ satisfaz $C \subset \overline{O^{+}(\eta)}, \forall \eta \in C$, provaremos o seguinte:

$$
M \backslash\{(t, y): t \in \mathbb{Z} ; y \in \mathbb{R}\} \subset O^{+}(\eta)
$$

Tomando o fecho em ambos os lados, temos $\overline{M \backslash\{(t, y): t \in \mathbb{Z}, y \in \mathbb{R}\}} \subset \overline{O^{+}(\eta)}$. Portanto $C \subset \overline{O^{+}(\eta)}$. 
Seja agora $\mathcal{F}=\{(t, y): t \in \mathbb{Z} . y \in \mathbb{R}\}$. Primeiro observamos que como $|\operatorname{sen} \pi(x+1)|=|\operatorname{sen}(\pi x)|$. se aplicamos para qualquer $(x, y)$ uma seqüencia de controles da forma

$$
\begin{aligned}
& u_{k}=0 \\
& v_{k}=\operatorname{sign}(g(x+k-1))
\end{aligned}
$$

Então, depois de $t$ passos, atingimos o ponto

$$
\left(x_{k}, y_{k}\right)=\left(x+t ; y+\frac{|\operatorname{sen} \pi x|}{2 \pi} \sum_{k=0}^{t-1} \frac{1}{x+k}\right) .
$$

Usando este fato e a divergência da série $\sum_{n} \frac{1}{n}$, provaremos o que queríamos. Fixemos $\left(x^{\prime}, y^{\prime}\right) \in C$ e $(\widehat{x}, \widehat{y}) \in M \backslash \mathcal{F}$. Observe que, como $\left(x^{\prime}, y^{\prime}\right) \notin C_{1}$, não existe restrição em assumir

$$
g\left(x^{\prime}\right) \neq 0 \quad \text { e } \quad y^{\prime} \neq 0 .
$$

Agora escolhemos $u_{k}, v_{k}$ como em (3.1). Como $g\left(x^{\prime}\right) \neq 0$, então existe $t$ tal que $y_{t}>1$. Logo, aplicamos uma seqüência de controles com todo $v_{k}=0$, até atingir o estado $(\bar{x}, \bar{y})$ do tipo:

$$
\begin{aligned}
& \bar{x}=\widehat{x}-n \\
& \bar{y}=y_{t},
\end{aligned}
$$

onde $n \in \mathbb{N}$ será escolhido depois. Observe que podemos assumir $\widehat{y}<\bar{y}$. Agora queremos achar uma seqüência de controles $\left(0, v_{k}\right)$ tais que obtemos o estado $(\widehat{x}, \widehat{y})$ em exatamente $n$ passos, é claro que isto é possível se e somente se

$$
\bar{y}-\frac{|\operatorname{sen} \pi \widehat{x}|}{2 \pi} \sum_{k=0}^{n-1} \frac{1}{|\widehat{x}-n+k|} \leq \widehat{y} .
$$

Assim, temos que escolher $n$ suficientemente grande tal que a desigualdade anterior é satisfeita. Isto é possível, pois

$$
\begin{aligned}
& \operatorname{sen}(\pi \widehat{x}) \neq 0 \quad \mathrm{e} \\
& \sum_{k=0}^{n-1} \frac{1}{|\widehat{x}-n+k|}=\sum_{m=1}^{n} \frac{1}{|\widehat{x}-m|} \quad \text { é divergente. }
\end{aligned}
$$


Portanto, $C$ é conjunto controlável.

Notemos que para este conjunto controlável $C$, núcleo de $C . N(C) \subset C=\operatorname{int} C$.

De fato, nenhum dos pontos do tipo $(-t, 0)$, com $t \in \mathbb{N}$, pertencem ao $N(C)$. mas se consideramos o correspondente conjunto transitivo maximal $\mathcal{C}$, é claro que $\mathcal{C}=\mathcal{U} \times M$. Assim, neste caso, a igualdade no Teorema 3.2 .3 é uma inclusão própria, isto é, int $C \subset \operatorname{int} \pi_{M} \mathcal{C}$.

\subsection{Decomposição de $\mathcal{U} \times M$ em componentes caóti- $\cos$}

Nesta seção final vamos discutir sistemas dinâmicos conhecidos como Axioma A, por duas razões principais:

- A decomposição de $\mathcal{U} \times M$ em componentes caóticos é semelhante à decomposição espectral de Smale [14].

- Os sistemas Axioma A são caóticos por partes. Então para determinar os conjuntos controláveis nestes conjuntos básicos uma idéia seria mergulhar a equação diferencial ordinária $\dot{x}=X_{0}(x)$ Axioma $\mathrm{A}$ (estruturalmente estável) no sistema de controle (2.1) para um controle dado.

Na parte final do Capítulo 1, quando definimos os difeomorfismos de Anosov, já falamos sobre a estrutura hiperbólica, mas agora vamos estabelecer seu análogo para fluxos.

Um conjunto invariante $\Lambda$ é hiperbólico para $\Phi_{t}$ (ou tem uma estrutura hiperbólica) se para cada $x \in \Lambda$ o espaço tangente $T M_{x}$ se decompõe em dois subespaços lineares $E_{x}^{s}$, $E_{x}^{u}$ tal que

(i) $T \Phi\left(E_{x}^{s, u}\right)=E_{\Phi(x)}^{s, u}$;

(ii) $\exists c, \lambda>0$ tal que $\left\|D \Phi_{t}(v)\right\| \leq c e^{-\lambda t}, \forall v \in E_{x}^{s} \mathrm{e}$ 
$\exists c_{1}, \mu>0$ tal que $\left\|D \Phi_{t}(u)\right\| \geq c_{1} e^{-\mu t} . \forall u \in E_{x}^{u}$

(iii) os subespaços $E_{x}^{s}, E_{x}^{u}$ dependem continuamente em $x \in \Lambda$.

Na seção 1.1 observamos que os pontos fixo e órbitas periódicas são conjuntos invaiiantes, que freqüentemente aparecem para atrair ou repelir as órbitas de pontos não contidas neles, ainda são minimais. O resultado seguinte cujo conjunto não errante $\Omega$ que tem uma estrutura hiperbólica dá as bases teóricas para as observações assinaladas.

Seja $\Phi_{t}(\phi): M \rightarrow M$ um fluxo (difeomorfismo) em variedade compacta sem bordo. com o conjunto não errante $\Omega\left(\Phi_{t}\right)=\Lambda \cup F$, onde:

- F é um conjunto finito de pontos fixos hiperbólicos de $\Phi_{t}$;

- $\Lambda$ é o fecho do conjunto de órbitas fechadas, que é hiperbólico por $\Phi_{t}$;

- $\Lambda \cap F=\emptyset$

(um conjunto não errante $\Omega(\phi)$ hiperbólico e os pontos periódicos de $\phi$ são densos em $\Omega(\phi))$.

Então $\Omega\left(\Phi_{t}\right)(\Omega(\phi))$ pode ser escrito como a união de um número finito de conjuntos básicos $\Omega_{i}$, isto é, $\Omega=\Omega_{1} \cup \cdots \cup \Omega_{k}$, onde cada $\Omega_{i}$ é fechado, invariante e contém uma órbita densa de $\Phi_{t}(\phi)$. Além do mais, a decomposição de $\Omega$ em conjuntos básicos é único e $M$ pode ser decomposto como a união disjunta $M=\bigcup_{i=0}^{m} W^{s}\left(\Omega_{i}\right)$, onde cada

$$
W^{s}\left(\Omega_{i}\right)=\left\{x \in M: \Phi_{t}(x) \rightarrow \Omega_{i}, \quad t \rightarrow \infty\right\}
$$

Fluxos (difeomorfismos) com conjunto não errante $\Omega$ que satisfazem as hipóteses anteriores são usualmente referidos como Axioma A, ver Smale [14]. Claramente, qualquer sistema dinâmico cujo conjunto não errante consiste de um número finito de pontos fixos ou órbitas periódicas é Axioma A. Além disso, pontos fixos e órbitas periódicas são conjuntos fechados, invariantes que trivialmente contêm uma órbita densa; isto é, são conjuntos 
básicos. Além disso, a equação (3.2) diz que cada $x \in M$ pertence ao final ou ao início de

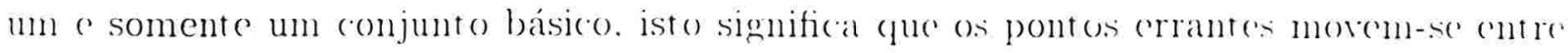
os conjuntos básicos. aproximando assintoticamente aqueles que são atratores. Alguns exemplos são ilustrados nas figuras seguintes.

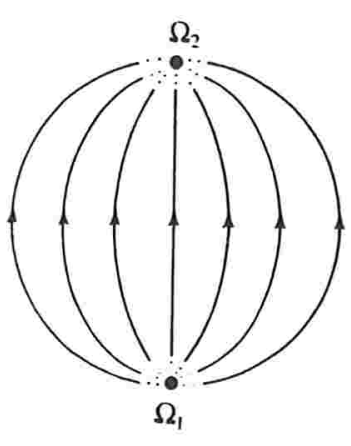

(a) $\quad \Omega_{1} \longrightarrow \Omega_{2}$

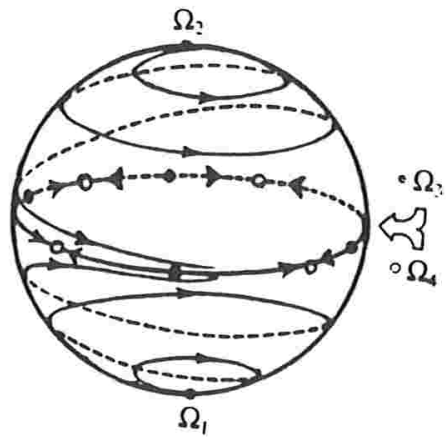

(b)

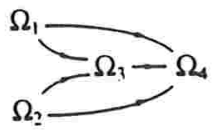

$\Omega$
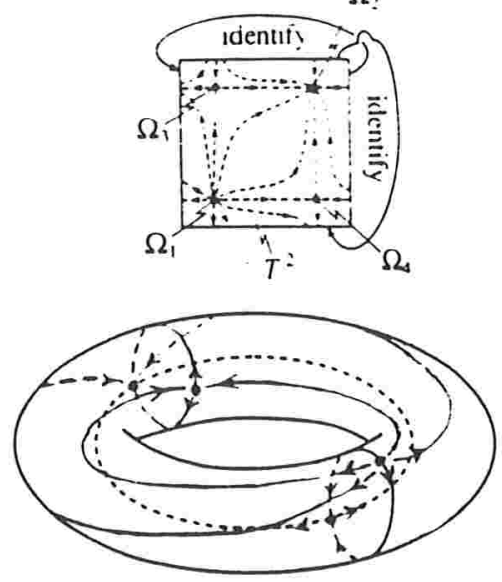

(c)<smiles>C1[Te]2O[Te]12</smiles>

Figura 3.1: Retrato de fase de campos axioma A em variedades compactas sem bordo

(a) Seja o fluxo $\Phi: S^{2} \rightarrow S^{2}$ que tem conjunto não errante $\Omega$ consistente de dois conjuntos básicos $\Omega_{1}$ e $\Omega_{2}$, ambos singularidades, todos os pontos errantes têm conjunto $\alpha$-limite $\Omega_{1}$ e conjunto $w$-limite $\Omega_{2}$. (b) Os conjuntos básicos $\Omega_{1}$ e $\Omega_{2}$ são singularidades instáveis. $\Omega_{3}$ são selas e $\Omega_{4}$ são estáveis. (c) Agora seja o fluxo $\Phi: T^{2} \rightarrow T^{2}$ os conjuntos básicos são todos singularidades: $\Omega_{1}$ é instável, $\Omega_{2}$ é estável e $\Omega_{3}, \Omega_{4}$ são tipo sela. Também é mostrada a dinâmica dos pontos errantes.

Agora vamos comentar os exemplos do Capítulo 1.

O difeomorfismo ferradura $\phi$, na esfera, é um exemplo importante de Axioma A. O conjunto não errante $\Omega$, deste difeomorfismo consiste de um conjunto invariante $\Lambda$ e dois pontos fixos; um estável e outro instável $\left(\Omega_{1}\right.$ instável. $\Omega_{2}$ estável). $\Omega$ tem uma estrutura hiperbólica e os pontos periódicos de $\Phi$ são densos em $\Omega$. então a decomposição espectral 
se aplica. isto é. $\Omega=\Omega_{1} \cup \Omega_{2} \cup \Lambda$. obviamente cada ponto fixo é um conjunto básico e mostramos que $\Lambda$ contém uma órbita densa de 0 , isto significa que uma decomposição adicional de $\Lambda$ está fora da questão, ele também é fechado (é um conjunto de Cantor) e invariante, então a única prossibilidade é que $\Omega_{3}=\Lambda$.

O difeomorfismo de Anosov dá uma outra ilustração da decomposição espectral, lembrando que os pontos periódicos deste difeomorfismo são densos e o conjunto não errante é todo o toro. Além disso, com $L=\left(\begin{array}{ll}2 & 1 \\ 1 & 1\end{array}\right)$ é claro que $E_{x}^{s}$ e $E_{x}^{u}$ são dados por $\left(1,\left(1-5^{1 / 2}\right) / 2\right)$ e $\left(1,\left(1+5^{1 / 2}\right) / 2\right)$, respectivamente, em cada ponto periódico $x$.

A continuidade determina que este deve ser assim para cada $x \in \Omega$, pois os pontos periódicos são densos. A decomposição do espaço tangente é portanto trivialmente contínua. Será o mesmo em cada ponto de $\Omega$ e como $L$ é hiperbólico, então satisfazem à contração e expansão em cada ponto, portanto $\Omega$ tem uma estrutura hiperbólica. Neste caso existe somente um conjunto básico $\Omega_{1}=\Omega=T^{2}$ e segue da decomposição espectral que o difeomorfismo de Anosov deve ter uma órbita que é densa no toro $T^{2}$. Ainda os sistemas estudados acima são abertos e densos [16].

Estes sistemas são o caso trivial na teoria de perturbações por sua estabilidade estrutural. Então cada conjunto básico pode ser aproximado por uma seqüência de conjuntos controláveis com $\operatorname{int} C_{i} \neq \emptyset, \forall 1 \leq i \leq n$; quando o controle tende a zero, sob hipóteses suficientemente ricas do (2.1). Assim, estudando o comportamento de seus conjuntos limites são determinados os conjuntos controláveis nesse tipo de sistemas. Aqui as demonstrações não são feitas; para maiores detalhes vide [6].

Vamos também dizer algumas palavras sobre a quantidade de conjuntos controláveis. Ele é um problema em aberto, mas já existem alguns resultados parciais, para casos particulares. No contexto de sistemas de controle semilinear que atuam em espaços projetivos e a sua extensão ao caso discreto foram provados por Colonius - Kliemann [5] e Wirth [18], respectivamente, a existência de um número finito de conjuntos controláveis 
com int $C_{i} \neq \emptyset$. O mesmo resultado foi obtido para ações de semigrupos em espaços homogêneos por San Martin-Tonelli [13].

Teorema 3.4.1 Se assumimos a existência de $C_{1}, \ldots, C_{l}$ conjuntos controláveis com interior nâo vazio que satisfazem $1 \leq l \leq n$ e a propriedade de acessibilidade local para (2.1). o sistema dinâmico $(\mathcal{U} \times M, G, \Psi)$ tem exatamente $1 \leq l \leq n$ extensões. Portanto. $\mathcal{C}_{1}, \ldots, \mathcal{C}_{l}$ componentes transitivas maximais com $\pi_{M} \mathcal{C}_{i} \neq \emptyset$ e $\pi_{M} \mathcal{C}_{i}=\bar{C}_{i}, i=1, \ldots, l$.

Demonstração: O Teorema 3.2 .3 aplicado a cada $C_{i}, i=1, \ldots, l$, conjunto controlável mostra o resultado.

Este resultado pode ser visto como uma analogia da decomposição de conjuntos de pontos não errantes do sistema dinâmico Axioma $\mathrm{A}$, discutido acima em um número finito de conjuntos topologicamente transitivo maximais. 


\section{Bibliografia}

[1] Albertini, F. and Sontag, E.D., Some connections between chaotic dynamical systems and control systems; in Proc. European Control Conference, vol.1. Grenoble, July (1991), pp.158-163.

[2] — Discrete-time transitivity and accebility: analiytic systems; SIAM J. Control and Optimization, vol.31, n.6 (1993), pp.1599-1622.

[3] Brezis, H., Analisis funcional, Alianza Editorial, S.A., Madrid (1984).

[4] Colonius, F. and Kliemann, W., Some aspects of control systems as dynamical systems; J. of Dynamics and Differential Equations, 5 (1993), pp.469-494.

[5] L Lienar control semigroups acting on projective spaces, J. of Dynamics and Differential Equations 5 (1993), pp.495-528.

[6] _ Limit behaviour and genericity for nonlinear control system, J. of Differential Equations 109 (1994), pp.8-41.

[7] Davydov, A.A., Qualitative theory of control systems, Translations of Mathematical Monographs, 141. American Mathematical (1994).

[8] Devaney, R.L., An introduction to chaotic dynamical systems, Benjamin/Cummings, Menlo Park, California (1989).

[9] Engelking, R., General topology, PWN - Polish Scientific Publishers, Warzawa (1977).

[10] Isidori, A., Nonlinear control theory, 2.ed, Springer-Verlag, New York/Berlin (1989).

[11] Mañe, R., Ergodic theory and differentiable dynamics, Springer, New York (1987).

[12] San Martin, L. and Arnold, L., A control problem related to the Lyapunov spectrum of stochastic flows, Mat. Aplicada e Computacional 5 (1986), pp.31-64.

[13] San Martin, L.A.B. and P.A. Tonelli, Semigroup actions on homogeneous spaces, Semigroup Forum, 50, pp.59-88 (1995).

[14] Smale, S., Differentiable dynamical systems; Bull. Amer. Math. Soc., 73, pp.747817 (1967). 
[15] Sussmann, H.J. and Jurdjevic. V., Controllability of nonlinear systems. J. Differential Equations, 12, pp.95-116 (1972).

[16] Palis, J. and de Melo. W., Geometric theory of dynamical systems: an introduction. Springer-Verlag: New York, Heidelberg, Berlin (1982).

[17] Tonelli, P.A., Control sets on homogeneous spaces, Thesis, University of Bremen (1991).

[18] Wirth, F.R., Robust stability of discrete-time systems under time-varying perturbations, PhD Thesis, Bremen (1995). 